\title{
Pacific
}

Journal of

Mathematics

\section{GENERAL KAC-MOODY ALGEBRAS AND THE KAZHDAN-LUSZTIG CONJECTURE}

WAYNE L. NEIDHARDT 


\section{GENERAL KAC-MOODY ALGEBRAS AND THE KAZHDAN-LUSZTIG CONJECTURE}

\section{WAYNE NEIDHARDT}

Let $\mathfrak{g}$ be a Kac-Moody algebra defined by a not necessarily symmetrizable generalized Cartan matrix. We use operators of coherent continuation to define modules $U_{\alpha} L(w \cdot \lambda)$ with $\alpha$ a simple root of $\mathfrak{g}$ and $w$ in the Weyl group $W$ of $\mathfrak{g}$, and then use these modules to study the integers $\operatorname{dim} \operatorname{Ext}^{n}(M(x \cdot \lambda), L(y \cdot \lambda))$ for $x$ and $y$ in $W$, where $\lambda$ is a dominant integral weight, $M(\mu)$ denotes the Verma module over $\mathfrak{g}$ of highest weight $\mu$ and $L(\mu)$ denotes its irreducible quotient. In particular, we show that in the presence of a parity conjecture and a weak assumption on the behavior of the modules $U_{\alpha} L(w \cdot \lambda)$, both of which hold in the case of a finite dimensional $\mathfrak{g}$, we may compute the dimensions by induction on the length of $x$, recovering the coefficients of "twisted" versions of the Kazhdan-Lusztig polynomials, where the twist comes from the fact that we start at the top of the orbit $W \cdot \lambda$, rather than at the bottom.

1. Introduction. Let $\mathfrak{g}$ be a Kac-Moody algebra over a field $K$ of characteristic zero defined by a not necessarily symmetrizable generalized Cartan matrix. Translation functors over $\mathfrak{g}$ were introduced in [6] and [7]. In the latter, operators of coherent continuation were introduced, but did not possess the desired self-adjointness which is so useful in the finite dimensional case, i.e. where $\mathfrak{g}$ is finite dimensional.

In the present work, we modify the definition of the translation functors somewhat, so that when we compose two translation functors to obtain an operator of coherent continuation, we do not begin and end in the same Weyl group orbit. We do, however, obtain two operators of coherent continuation which are adjoint to each other. We then use these operators of coherent continuation to define modules $U_{\alpha} L(w \cdot \lambda)$ with $\alpha$ a simple root of $\mathfrak{g}$ and $w$ in the Weyl group $W$ of $\mathfrak{g}$, and use these modules to study the integers $\operatorname{dim} \operatorname{Ext}_{C(\lambda)}^{n}(M(x \cdot \lambda), L(y \cdot \lambda))$ for $x$ and $y$ in $W$, where $\lambda$ is a dominant integral weight, $C(\lambda)$ denotes the category of weight modules all of whose weights are less than or equal to $\lambda, M(\mu)$ denotes the Verma module over $\mathfrak{g}$ of highest weight $\mu$ and $L(\mu)$ denotes its irreducible quotient.

In our study of these dimensions, we show that the various 
$\operatorname{Ext}_{C(\lambda)}^{n}(M(x \cdot \lambda), L(y \cdot \lambda))$ occur as terms in long exact sequences involving the modules $U_{\alpha} L(w \cdot \lambda)$, just as they do in the finite dimensional case. In particular, we show that in the presence of a parity conjecture and a weak assumption on the behavior of the modules $U_{\alpha} L(w \cdot \lambda)$, both of which hold in the case of a finite dimensional $\mathfrak{g}$, we may compute the dimensions by induction on the length of $x$, recovering the coefficients of "twisted" versions of the Kazhdan-Lusztig polynomials, where the twist comes from the fact that we start at the top of the orbit $W \cdot \lambda$, rather than at the bottom.

It is worth noting that the major accomplishment in this work is not the reduction of the computation of the integers

$$
\operatorname{dim} \operatorname{Ext}_{C(\lambda)}^{n}(M(x \cdot \lambda), L(y \cdot \lambda))
$$

to these conjectures, but rather the definition itself of the modules $U_{\alpha} L(w \cdot \lambda)$. Since, in the nonsymmetrizable case, we do not have the Casimir operator and can possibly have Verma module imbeddings between modules of highest weights in different Weyl group orbits, considerable care must be taken just to define the translation functors. Also, in defining the translation functors in one direction we are tensoring with an infinite dimensional highest weight module, which means that the translation functor in the reverse direction involves tensoring with an infinite dimensional lowest weight module. Thus, when attempting to show adjointness, these two functors are not interchangeable; one must be the left adjoint and the other the right. For this reason, in order for the operators of coherent continuation to have nice adjoint properties, we cannot simply return to the same orbit. More precisely, to obtain an operator of coherent continuation, we translate from the orbit of a dominant integral weight $\lambda$ to an integral weight $\lambda_{\alpha}$ on the $\alpha$-wall by tensoring with a highest weight module, and then translate from $\lambda_{\alpha}$ to a different dominant integral weight $\mu$ by tensoring with a highest weight module. The operator of coherent continuation in the reverse direction then translates from $\mu$ to $\lambda_{\alpha}$ to $\lambda$ by tensoring with lowest weight modules at each stage. Further difficulties are encountered as we try to control the composition factors of highest weights in the other orbits under translation. We would like to have "lower" orbits remain lower and "higher" orbits remain higher, but this cannot be completely controlled. For this reason, we work only with $\lambda$ and $\mu$ close enough to the $\alpha$-wall that only two orbits become reversed under translation, and these orbits can be sorted out by the representation theory of $\operatorname{sl}(2, K)$. Thus the mere 
fact that we can define $U_{\alpha} L(w \cdot \lambda)$ may be viewed as the significant result of the present paper.

In $\S 2$ we give the definitions, notation, and background associated with Kac-Moody algebras. Section 3 generalizes some previous results from [7] on Verma series and reverse Verma series to modules with highest weight series and reverse highest weight series. In $\S 4$ we define certain functors on modules with highest weight series and reverse highest weight series which essentially pick out those factors with highest weights in a particular set of weights, such as a Weyl group orbit. We also show that these functors act as adjoints to certain functors previously defined in [6]. In $\S 5$, we define the translation functors and operators of coherent continuation and prove their adjoint properties. The behavior of Verma modules and irreducible highest weight modules under translation and coherent continuation is studied in $\S 6$, resulting in the definition of $U_{\alpha} L(w \cdot \lambda)$. Finally, in $\S 7$ we apply this to study the integers $\operatorname{dim} \operatorname{Ext}_{C(\lambda)}^{n}(M(x \cdot \lambda), L(y \cdot \lambda))$.

2. Notation and background. In this section we give the basic definitions and notation associated with Kac-Moody algebras and their representations, and introduce certain categories of modules and filtrations of modules. We also state some basic results which will be needed in the sequel.

Let $A=\left(A_{i j}\right)$ be an $l \times l$ generalized Cartan matrix, meaning that $A_{i i}=2$ for all $i, A_{i j}$ is a nonpositive integer for $i \neq j$, and $A_{i j}=0$ if and only if $A_{j i}=0$. Let $K$ be a field of characteristic zero, and let $\mathfrak{g}$ be the Kac-Moody Lie algebra over $K$ defined by $A$, so that $\mathfrak{g}$ satisfies

(i) $\mathfrak{g}$ is generated by an abelian subalgebra $\mathfrak{h}$, called the Cartan subalgebra and satisfying $\operatorname{dim}_{K} \mathfrak{h}=l+\operatorname{corank}(A)$, together with elements $e_{1}, \ldots, e_{l}, f_{1}, \ldots, f_{l}$, called simple root vectors and negative simple root vectors, respectively.

(ii) There are linearly independent sets $\left\{h_{1}, \ldots, h_{l}\right\}$ in $\mathfrak{h}$ and $\left\{\alpha_{1}, \ldots, \alpha_{l}\right\}$ in $\mathfrak{h}^{*}$ such that $A_{i j}=\alpha_{j}\left(h_{i}\right)$ for all $i$ and $j$. The $\alpha_{i}$ 's are called the simple roots, and the $h_{i}$ 's are called the simple coroots.

(iii) $\left[e_{i}, f_{j}\right]=\delta_{i j} h_{i}$ for all $i, j=1, \ldots, l$.

(iv) $\left[h, e_{i}\right]=\alpha_{i}(h) e_{i}$ and $\left[h, f_{i}\right]=-\alpha_{i}(h) f_{i}$ for all $h \in \mathfrak{h}$ and all $i=1, \ldots, l$.

(v) $\left(\operatorname{ad} e_{i}\right)^{-A_{i j}+1}\left(e_{j}\right)=0=\left(\operatorname{ad} f_{i}\right)^{-A_{i j}+1}\left(f_{j}\right)$ for all $i \neq j$.

(vi) There is an involutive antiautomorphism $\sigma: \mathfrak{g} \rightarrow \mathfrak{g}$ such that $\sigma\left(e_{i}\right)=f_{i}$ for all $i=1, \ldots, l$, and $\sigma(h)=h$ for all $h \in \mathfrak{h}$. 
Denote by $\mathfrak{n}^{+}$(respectively, $\mathfrak{n}^{-}$) the subalgebra of $\mathfrak{g}$ generated by $\left\{e_{1}, \ldots, e_{l}\right\}$ (respectively, $\left\{f_{1}, \ldots, f_{l}\right\}$ ), and set $\mathfrak{b}=\mathfrak{h} \oplus \mathfrak{n}^{+}$, called the Borel subalgebra of $\mathfrak{g}$. For each $i=1, \ldots, l$, let $\mathfrak{a}_{i}=$ $K h_{i} \oplus K e_{i} \oplus K f_{i}$, a subalgebra of $\mathfrak{g}$ isomorphic to $\mathrm{sl}(2, K)$.

For any $\mathfrak{h}$-module $M$ and any $\lambda \in \mathfrak{h}^{*}$, let $M_{\lambda}=\{m \in M \mid h \cdot m=$ $\lambda(h) m$ for all $\left.h \in \mathfrak{h}^{*}\right\}$, called the $\lambda$-weight space of $M$; if $M_{\lambda} \neq 0$ we call $\lambda$ a weight of $M$. Note that this notion of weights applies as well to $\mathfrak{g}$-modules and $\mathfrak{b}$-modules, which may be viewed as $\mathfrak{h}$-modules by restriction. In particular, for the adjoint representation of $\mathfrak{g}$, we let $\Delta=\left\{\alpha \in \mathfrak{h}^{*} \mid g_{\alpha} \neq 0\right.$ and $\left.\alpha \neq 0\right\}$ and call $\Delta$ the set of roots of $\mathfrak{g}$, and call each $\mathfrak{g}_{\alpha}$ with $\alpha \in \Delta$ the $\alpha$-root space of $\mathfrak{g}$. In case $M$ is a $g$-module satisfying $M=\bigoplus_{\lambda \in \mathfrak{h}^{*}} M_{\lambda}$, we call $M$ a weight module, and write $\Pi(M)$ for the set of weights of $M$. If in addition each $M_{\lambda}$ is finite dimensional, we define the character of $M$ to be $\operatorname{ch} M=\sum_{\lambda \in h^{*}}\left(\operatorname{dim} M_{\lambda}\right) e^{\lambda}$, where the $e^{\lambda}$ 's are formal exponentials.

In the sequel, whenever $M$ and $N$ are weight modules, we will write $\operatorname{Hom}(M, N)$ for the set of $\mathfrak{g}$-module morphisms from $M$ to $N$, and $\operatorname{Ext}^{n}(M, N)$ for the set of equivalence classes of $n$-extensions of $M$ by $N$ in the category of weight modules. We also write $\operatorname{Ext}(M, N)$ for $\operatorname{Ext}^{1}(M, N)$.

The root lattice is $Q=\sum_{i=1}^{l} \mathbb{Z} \alpha_{j}$, and we let $Q^{+}=\sum_{i=1}^{l} \mathbb{Z}_{+} \alpha_{i}$, where $\mathbb{Z}_{+}$is the set of nonnegative integers. For any $\eta \in Q^{+}$, we define the height of $\eta$ to be $\mathrm{ht}(\eta)=\sum_{i=1}^{l} k_{i}$, where $\eta=\sum_{i=1}^{l} k_{i} \alpha_{i}$. We may define a partial order on $\mathfrak{h}^{*}$ by letting $\mu \leq \lambda$ if $\lambda-\mu \in Q^{+}$. We set $\Delta^{+}=\{\alpha \in \Delta \mid \alpha>0\}$, called the set of positive roots, and $\Delta^{-}=-\Delta^{+}=\{\alpha \in \Delta \mid \alpha<0\}$. Also, let $P=\left\{\lambda \in \mathfrak{h}^{*} \mid \lambda\left(h_{i}\right) \in \mathbb{Z}\right.$ for all $i\}$, called the set of integral weights, and let $P^{+}=\left\{\lambda \in \mathfrak{h}^{*} \mid \lambda\left(h_{i}\right) \in \mathbb{Z}_{+}\right.$ for all $i$, called the set of dominant integral weights.

For any $\lambda \in \mathfrak{h}^{*}$, we denote by $C(\lambda)$ the full subcategory of the category of $\mathfrak{g}$-modules $M$ such that $M$ is a weight module with finite dimensional weight spaces and such that $\Pi(M) \subseteq\{\mu \mid \mu \leq \lambda\}$.

Suppose $M$ is a $\mathfrak{g}$-module. If $v \in M_{\lambda}$ is a nonzero vector satisfying $\mathfrak{n}^{+} \cdot v=0$, we call $v$ a maximal vector. If in addition $M=U(\mathfrak{g}) v$, where $U(-)$ denotes the universal enveloping algebra functor, we call $v$ a highest weight vector and $M$ a highest weight module of weight $\lambda$. Every highest weight module is a weight module.

Let $\lambda \in \mathfrak{h}^{*}$, and denote by $K(\lambda)$ the one-dimensional $\mathfrak{b}$-module of weight $\lambda$ on which $\mathfrak{n}^{+}$acts trivially. The Verma module of highest weight $\lambda$ is then the induced $\mathfrak{g}$-module $M(\lambda)=U(\mathfrak{g}) \otimes_{U(\mathfrak{b})} K(\lambda)$. It is well known that $M(\lambda)$ is the universal highest weight module 
of weight $\lambda$, and that it has a unique irreducible quotient, which is denoted $L(\lambda)$. Similarly, we may define lowest weight modules, and let $W(\lambda)$ be the universal lowest weight module of weight $\lambda$ and $\Gamma(\lambda)$ its unique irreducible quotient. (These latter two modules may be viewed as "upside-down" versions of $M(\lambda)$ and $L(\lambda)$; hence the choice of notation.) If $\lambda, \mu \in \mathfrak{h}^{*}$, it is easy to see that any nonzero morphism from $M(\lambda)$ to $M(\mu)$ must be injective.

The Weyl group $W$ is defined as follows. For each $i=1, \ldots, l$, define the linear involution $s_{i}: \mathfrak{h}^{*} \rightarrow \mathfrak{h}^{*}$ by $s_{i}(\lambda)=\lambda-\lambda\left(h_{i}\right) \alpha_{i}$, and take $W$ to be the subgroup of $\mathrm{GL}\left(\mathfrak{h}^{*}\right)$ generated by $\left\{s_{1}, \ldots, s_{l}\right\}$. For any $w \in W$, define the length of $w$, denoted $l(w)$, to be the smallest integer $n$ such that $w$ is expressible in the form $s_{i_{1}} \cdots s_{i_{n}}$ for some $i_{1}, \ldots, i_{n} \in\{1, \ldots, l\}$. Let $\rho \in \mathfrak{h}^{*}$ be a fixed element such that $\rho\left(h_{i}\right)=1$ for all $i$, and define the dot action of $W$ on $\mathfrak{h}^{*}$ by $w \cdot \lambda=w(\lambda+\rho)-\rho$.

If $L$ is a weight module which is a direct sum of finite dimensional $\left(\mathfrak{a}_{i}+\mathfrak{h}\right)$-modules for each $i=1, \ldots, l$, we say that $L$ is integrable. It is well known, and easy to see from the representation theory of $\operatorname{sl}(2, K)$, that for any integrable $g$-module $L$, any $\lambda \in \mathfrak{h}^{*}$, and any $w \in W$, we have $\operatorname{dim} L_{\lambda}=\operatorname{dim} L_{w \lambda}$. It is also easy to see that when $\eta \in P^{+}$, both $L(\eta)$ and $\Gamma(-\eta)$ are integrable.

Certain types of filtrations of modules will be useful in the sequel.

Definition 2.1 ([2]). Let $M$ be a weight module, all of whose weight spaces are finite dimensional, and let $\mu \in \mathfrak{h}^{*}$. By a local composition series (LCS) for $M$ at $\mu$, we mean a finite sequence $0=M_{0} \subset M_{1} \subset \cdots \subset M_{n}=M$ of submodules of $M$ such that each factor $F_{i}=M_{i} / M_{i-1}$ satisfies either $\Pi\left(F_{i}\right) \cap\{\nu \mid \nu \geq \mu\}=\varnothing$ or $F_{i} \cong L\left(\mu_{i}\right)$ for some $\mu_{i} \geq \mu$. In case $M$ has such an LCS, we write $(M: L(\mu))$ for the number of $i$ such that $\mu_{i}=\mu$. If $(M: L(\mu)) \neq 0$, we call $L(\mu)$ a composition factor of $M$.

Remark. In [2, Proposition 4.2] it is shown that if $\sum_{\nu \geq \mu} \operatorname{dim} M_{\nu}$ is finite, then $M$ has an LCS at $\mu$. In particular, such series always exist for modules which are objects in categories of the form $C(\lambda)$. It is also shown in [2] that the multiplicity $(M: L(\mu))$ does not depend on the choice of LCS. One also obtains the same multiplicities, when ch $M$ exists, by defining these multiplicities by $\operatorname{ch} M=$ $\sum_{\mu \in \mathfrak{h}^{*}}(M: L(\mu)) \operatorname{ch} L(\mu)$.

Definition 2.2. Let $M$ be a weight module. By a Verma series (VS) for $M$ we mean a sequence (possibly finite) $0=M_{0} \subset M_{1} \subset \cdots$ 
of submodules of $M$ such that (i) $M=\bigcup_{i \geq 0} M_{i}$, (ii) each factor $F_{i}=$ $M_{i} / M_{i-1} \cong M\left(\lambda_{i}\right)$ for some $\lambda_{i} \in \mathfrak{h}^{*}$, and (iii) for any $\mu \in \mathfrak{h}^{*},\left\{i \mid \lambda_{i} \geq\right.$ $\mu\}$ is a finite set. In case $M$ has such a VS and $\mu \in \mathfrak{h}^{*}$, we write $[M: M(\mu)]$ for the number of $i$ such that $\lambda_{i}=\mu$. If $[M: M(\mu)] \neq 0$, we call $M(\mu)$ a Verma factor of $M$.

REMARK. Condition (iii) above is equivalent to the requirement that all weight spaces of $M$ be finite dimensional, but compare this with Definition 2.3 below. Also, the numbers $[M: M(\mu)]$, when they are defined, can easily be seen to be independent of the choice of VS, by character considerations. In fact, we obtain the same result by using ch $M=\sum_{\mu \in \mathfrak{h}^{*}}[M: M(\mu)] \operatorname{ch} M(\mu)$ to define the multiplicities, when $M$ has a VS. For this reason, we can and do use this formula to define the multiplicities $[M: M(\mu)]$ whenever $M$ is an object in one of the categories of the form $C(\lambda)$, but not necessarily with a VS.

Definition 2.3. Let $M$ be a weight module. By a reverse Verma series (RVS) for $M$, we mean a sequence (possibly finite) $M=M_{0} \supset$ $M_{1} \supset \cdots$ of submodules of $M$ such that (i) $\bigcap_{i \geq 0} M_{i}=0$, (ii) each factor $F_{i}=M_{i-1} / M_{i} \cong M\left(\lambda_{i}\right)$ for some $\lambda_{i} \in \mathfrak{h}^{*}$, (iii) for any $\mu \in \mathfrak{h}^{*}$, $\left\{i \mid \lambda_{i} \leq \mu\right\}$ is a finite set, and (iv) there exist weight vectors $v_{i} \in M_{\lambda_{1}}$ such that $v_{i}+M_{i}$ is a highest weight vector for $F_{i}$ for each $i=$ $1,2, \ldots$, and such that $M_{i}=\sum_{j>i} U\left(\mathfrak{n}^{-}\right) v_{j}$ for each $i=0,1, \ldots$. In case $M$ has such an RVS and $\mu \in \mathfrak{h}^{*}$, we write $[M: M(\mu)]$ for the number of $i$ such that $\lambda_{i}=\mu$. If $[M: M(\mu)] \neq 0$, we call $M(\mu)$ a Verma factor of $M$.

REMARK. The idea of an RVS was introduced in [7], where it was shown that the numbers $[M: M(\mu)]$, when they are defined, are independent of the choice of RVS.

The following proposition, which is easy to prove, deals with some cases in which VS and RVS actually occur.

Proposition 2.4 ([7, Propositions 4.16 and 4.17]). (i) Suppose that $M$ has a VS and that $N$ is a highest weight module with highest weight $\mu$. Then $M \otimes_{K} N$ also has a VS, and $\left[M \otimes_{K} N: M(\nu)\right]=$ $\sum_{\chi \leq \mu}[M: M(\nu-\chi)] \operatorname{dim} N_{\chi}$ for any $\nu \in \mathfrak{h}^{*}$. In particular, $M(\lambda) \otimes_{K}$ $L(\mu)$ has a VS for any $\lambda, \mu \in \mathfrak{h}^{*}$.

(ii) Suppose that $M$ has an RVS and that $N$ is a lowest weight module with lowest weight $\mu$. Then $M \otimes_{K} N$ also has an RVS, and $\left[M \otimes_{K} N: M(\nu)\right]=\sum_{\chi \geq \mu}[M: M(\nu-\chi)] \operatorname{dim} N_{\chi}$ for any $\nu \in \mathfrak{h}^{*}$. In particular, $M(\lambda) \otimes_{K} \Gamma(\mu)$ has an $R V S$ for any $\lambda, \mu \in \mathfrak{h}^{*}$. 
In a similar vein, we also define the concepts of highest weight series and reverse highest weight series.

Definition 2.5. Let $M$ be a weight module. By a highest weight series (HWS) for $M$ we mean a sequence (possibly finite) $0=M_{0} \subset$ $M_{1} \subset \cdots$ of submodules of $M$ such that (i) $M=\bigcup_{i \geq 0} M_{i}$, (ii) each factor $F_{i}=M_{i} / M_{i-1}$ is a highest weight module of weight $\lambda_{i}$ for some $\lambda_{i} \in \mathfrak{h}^{*}$, and (iii) for any $\mu \in \mathfrak{h}^{*},\left\{i \mid \lambda_{i} \geq \mu\right\}$ is a finite set.

REMARK. The concept of highest weight series was introduced in [3], where it was shown that any module in a category of the form $C(\lambda)$ has an HWS. Also, condition (iii) above is equivalent to the requirement that all weight spaces of $M$ be finite dimensional, but compare this with Definition 2.6 below.

Definition 2.6. Let $M$ be a weight module. By a reverse highest weight series (RHWS) for $M$, we mean a sequence (possibly finite) $M=M_{0} \supset M_{1} \supset \cdots$ of submodules of $M$ such that (i) $\bigcap_{i \geq 0} M_{i}=0$, (ii) each factor $F_{i}=M_{i-1} / M_{i}$ is a highest weight module of weight $\lambda_{i}$ for some $\lambda_{i} \in \mathfrak{h}^{*}$, (iii) for any $\mu \in \mathfrak{h}^{*},\left\{i \mid \lambda_{i} \leq \mu\right\}$ is a finite set, and (iv) there exist weight vectors $v_{i} \in M_{\lambda_{i}}$ such that $v_{i}+M_{i}$ is a highest weight vector for $F_{i}$ for each $i=1,2, \ldots$, and such that $M_{i}=\sum_{j>i} U\left(\mathfrak{n}^{-}\right) v_{j}$ for each $i=0,1, \ldots$

We now recall the finitely generated indecomposable projective objects in $C(\lambda)$, for any fixed $\lambda \in \mathfrak{h}^{*}$, introduced in [8]. For any $\mu \leq \lambda$, let

$$
P^{\lambda}(\mu)=U(\mathfrak{g}) \otimes_{U(\mathfrak{b})}\left\{\left[U\left(\mathfrak{n}^{+}\right) /\left(\bigoplus_{\mu+\alpha \leq \lambda} U\left(\mathfrak{n}^{+}\right)_{\alpha}\right)\right] \otimes_{K} K(\mu)\right\},
$$

which is a projective object in $C(\lambda)$, as was shown in [8], since for any $M \in \mathrm{Ob} C(\lambda), \operatorname{Hom}\left(P^{\lambda}(\mu), M\right)$ is naturally isomorphic to $\operatorname{Hom}_{\mathfrak{h}}(K(\mu), M)$, and hence to $M_{\mu}$. Note that $P^{\lambda}(\mu)$ is finitely generated-in fact, it is generated by the weight vector $1 \otimes \overline{1} \otimes 1$ of weight $\mu$. The indecomposable summands of the various $P^{\lambda}(\mu)$ with $\mu \leq \lambda$ have several nice properties, which we summarize here.

Proposition 2.7 ([8, Propositions 4.5, 4.7, 4.8, and 5.3, Lemma 4.12, Corollary 4.13, and Theorem 6.2]). For each irreducible object $L(\mu)$ in $C(\lambda)$, there is a unique (up to isomorphism) finitely generated indecomposable projective object $I^{\lambda}(\mu)$ in $C(\lambda)$ which maps onto $L(\mu)$. Conversely, every finitely generated indecomposable projective object in $C(\lambda)$ has a unique irreducible quotient, so that $L(\mu) \leftrightarrow I^{\lambda}(\mu)$ 
gives a one-to-one correspondence between the irreducible objects in $C(\lambda)$ and the finitely generated indecomposable projective objects in $C(\lambda)$. Furthermore, $I^{\lambda}(\mu)$ has a finite VS with $M(\mu)$ as the top factor, and $\left[I^{\lambda}(\mu): M(\nu)\right]=(M(\nu): L(\mu))$ for all $\mu, \nu \leq \lambda$. Finally, for any $M \in \mathrm{Ob} C(\lambda)$ we have $(M: L(\mu))=\operatorname{dim} \operatorname{Hom}\left(I^{\lambda}(\mu), M\right)$.

It is easy to see that $I^{\lambda}(\mu)$ is a summand of $P^{\lambda}(\mu)$, so that $I^{\lambda}(\mu)$ is generated by a weight vector of weight $\mu$.

The following result, which follows easily from the fact that the modules $I^{\lambda}(\mu)$ have finite VS as specified in the above proposition, will be needed in the sequel.

Proposition 2.8 ([6, Theorem 3.5]). Let $\Gamma$ be the directed graph whose vertex set is $\mathfrak{h}^{*}$ and with an edge of multiplicity $(M(\mu): L(\nu))$ from $\nu$ to $\mu$ for each $\nu<\mu$. We denote by $g_{n}(\nu, \mu)$ the number of paths of length $n$ from $\mu$ to $\nu$ in $\Gamma$. Let $\lambda \in \mathfrak{h}^{*}$, and let $M \in \mathrm{Ob} C(\lambda)$ have a finite VS. Then $M$ has a projective resolution in $C(\lambda)$

$$
\ldots \stackrel{d_{n+1}}{\longrightarrow} P_{n} \stackrel{d_{n}}{\longrightarrow} \cdots \stackrel{d_{1}}{\longrightarrow} P_{0} \stackrel{d_{0}}{\longrightarrow} M \rightarrow 0
$$

such that each $P_{n}$ is a finite direct sum of various $I^{\lambda}(\nu)$ and such that each $\operatorname{Ker} d_{n}=\operatorname{Im} d_{n+1}$ has a finite VS. The projective resolution can be chosen so that for any $\nu \leq \lambda$ the multiplicity of $I^{\lambda}(\nu)$ as a summand of $P_{n}$ is equal to $\left[\operatorname{Im} d_{n}: M(\nu)\right]$, and both multiplicities are equal to $\sum_{\mu \leq \lambda}[M: M(\mu)] g_{n}(\nu, \mu)$. In particular, if all Verma factors $M(\mu)$ of $M$ satisfy $\mu \geq \chi$ for some $\chi \in \mathfrak{h}^{*}$, then the same is true of all Verma factors of each $\operatorname{Ker} d_{n}=\operatorname{Im} d_{n+1}$.

We make the observation for use in the sequel that it follows immediately from the above proposition that $\operatorname{Ext}_{C(\lambda)}^{n}(M(\mu), M)=0$ for all $n$ whenever $M$ has no weights $\geq \mu$.

We conclude this section with some facts relating Verma module imbeddings, composition factors, and extensions of Verma modules.

Proposition 2.9 ([5, Propositions 2.1 and 2.4] and [7, Proposition 4.6]). Let $\lambda, \mu, \nu \in \mathfrak{h}^{*}$ with $\mu, \nu \leq \lambda$. Then $\operatorname{Hom}(M(\nu), M(\mu)) \neq 0$ if and only if $(M(\mu): L(\nu)) \neq 0$. Also, if $M$ is a highest weight: module of weight $\mu$ and $\operatorname{Ext}_{C(\lambda)}^{n}(M(\nu), M) \neq 0$, then there exist weights $\nu=\nu_{n}<\nu_{n-1}<\cdots<\nu_{0} \leq \mu$ with $\left(M(\mu): L\left(\nu_{0}\right)\right) \neq 0$ and $\left(M\left(\nu_{i-1}\right): L\left(\nu_{i}\right)\right) \neq 0$ for all $i=1, \ldots, n$. In particular, if $\operatorname{Ext}(M(\nu), M) \neq 0$, then $\nu<\mu$ and $(M(\mu): L(\nu)) \neq 0$. 
3. Highest weight series and reverse highest weight series. In this section, we examine some properties of modules with HWS or RHWS. In particular, we describe how certain partitions of sets of weights induce corresponding filtrations on modules with HWS or RHWS, where the ordering of the factors can be controlled. We also give a criterion for distinguishing modules with RHWS. The results of this section essentially generalize results from [7] on modules with VS or RVS.

LEMMA 3.1. Let sets of weights $S_{1}$ and $S_{2}$ be given such that $\operatorname{Hom}(M(\lambda), M(\mu))=0$ whenever $\lambda \in S_{1}$ and $\mu \in S_{2}$. Suppose we have $\mathfrak{g}$-modules $N \subset M$, where $M / N$ is a highest weight module of weight $\lambda \in S_{1}$ and $N$ is a highest weight module of weight $\mu \in S_{2}$. Then there exists a submodule $N^{\prime}$ of $M$ such that $N^{\prime}$ is a highest weight module of weight $\lambda$, and either $M / N^{\prime}$ is a highest weight module of weight $\mu$ or $M / N^{\prime}=0$.

Proof. Note that if $v_{1}+N$ is any highest weight vector of $M / N$ with $v_{1} \in M_{\lambda}$ and if $v_{2}$ is any highest weight vector of $N$, then $M=U\left(\mathfrak{n}^{-}\right) v_{1}+U\left(\mathfrak{n}^{-}\right) v_{2}$. Observe that $\mathfrak{n}^{+} \cdot v_{2}=0$ for any such choice of $v_{1}$ and $v_{2}$. We show that $v_{1}$ may be chosen so that $\mathfrak{n}^{+} \cdot v_{1}=0$, also.

Let $\pi: M(\lambda) \rightarrow M / N$ be an epimorphism, and consider the pullback

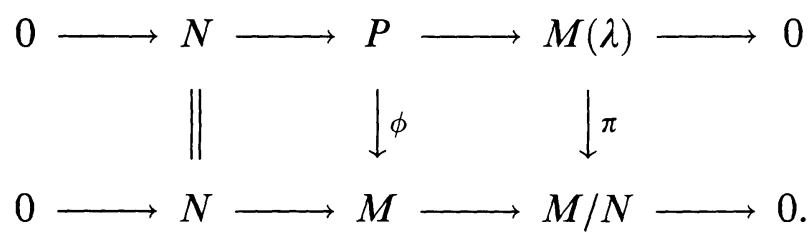

Since $\operatorname{Ext}(M(\lambda), N)=0$ by Proposition 2.9 and our hypotheses on $S_{1}$ and $S_{2}$, we must have $P \cong N \oplus M(\lambda)$. Identifying $M(\lambda)$ with its corresponding direct summand in $P$, and letting $v$ be a highest weight vector for $M(\lambda)$, we set $v_{1}=\phi(v)$. Then $\mathfrak{n}^{+} \cdot v_{1}=0, v_{1} \in M_{\lambda}$, and $v_{1}+N$ is a highest weight vector for $M / N$, by an easy diagram chase.

Finally, setting $N^{\prime}=U\left(\mathfrak{n}^{-}\right) v_{1}$ gives the result, since $M / N^{\prime}$ is generated by $v_{2}+N^{\prime}$.

This lemma will allow us to switch the order of the factors in a highest weight series or a reverse highest weight series without increasing the number of factors, thereby producing useful filtrations of modules. We begin by applying this to modules with RHWS. 
Proposition 3.2. Let sets of weights $S_{1}$ and $S_{2}$ be given such that $\operatorname{Hom}(M(\lambda), M(\mu))=0$ whenever $\lambda \in S_{1}$ and $\mu \in S_{2}$. Suppose $M$ has an RHWS $M=M_{0} \supset M_{1} \supset M_{2} \supset \cdots$ where all factors have highest weights in $S_{1} \cup S_{2}$, and such that only a finite number of factors have highest weights in $S_{2}$. Then there is a submodule $N$ of $M$ such that $N$ has an RHWS, all of whose factors have highest weights in $S_{1}$, and such that $M / N$ has a finite $R H W S$, all of whose factors have highest weights in $S_{2}$.

Proof. This follows by repeated application of Lemma 3.1 to each of the finitely many factors with highest weights in $S_{2}$, switching the order of the factors to bring them to the top of the RHWS or eliminate them.

Proposition 3.3. Let sets of weights $S_{1}$ and $S_{2}$ be given such that $\operatorname{Hom}(M(\lambda), M(\mu))=0$ whenever $\lambda \in S_{1}$ and $\mu \in S_{2}$. Suppose that $M$ and $M^{\prime}$ each satisfy the hypotheses of Proposition 3.2, and further suppose that $N \subset M$ and $N^{\prime} \subset M^{\prime}$ are submodules satisfying the conclusion of Proposition 3.2. Then for any $\phi \in \operatorname{Hom}\left(M, M^{\prime}\right)$, we must have $\phi(N) \subset N^{\prime}$, so that $\phi$ induces maps $\phi_{1}: N \rightarrow N^{\prime}$ and $\phi_{2}: M / N \rightarrow M^{\prime} / N^{\prime}$.

Proof. If this were not the case, then $\phi$ would induce a nonzero $\operatorname{map} \bar{\phi}: N \rightarrow M^{\prime} / N^{\prime}$. Let $N=N_{0} \supset N_{1} \supset N_{2} \supset \cdots$ be an RHWS for $N$, and choose weight vectors $v_{i} \in N$ in accordance with Definition $2.6(\mathrm{iv})$ for $i=1,2,3, \ldots$. Since $M^{\prime} / N^{\prime}$ has only a finite number of factors in its RHWS, the finiteness condition in Definition 2.6(iii) guarantees that $\bar{\phi}\left(v_{i}\right)=0$ for $i$ large enough. Thus, there is some $k$ with $\operatorname{Hom}\left(N / N_{k}, M^{\prime} / N^{\prime}\right) \neq 0$. But now each of $N / N_{k}$ and $M^{\prime} / N^{\prime}$ has only finitely many factors in its RHWS. We see that there is at least one factor $F$ of the RHWS for $N / N_{k}$, and hence of $N$, and at least one factor $F^{\prime}$ of the RHWS for $M^{\prime} / N^{\prime}$, such that $\operatorname{Hom}\left(F, F^{\prime}\right) \neq 0$. But $F$ has some highest weight $\lambda \in S_{1}$ and $F^{\prime}$ has some highest weight $\mu \in S_{2}$, which, by Proposition 2.9, implies that $\operatorname{Hom}(M(\lambda), M(\mu)) \neq 0$, contrary to our hypothesis on $S_{1}$ and $S_{2}$. Therefore $\phi(N) \subset N^{\prime}$.

Corollary 3.4. Let $S_{1}, S_{2}$, and $M$ satisfy the hypotheses of Proposition 3.2. Then the submodule $N$ given in the conclusion of Proposition 3.2 is unique. 
Proof. This follows immediately from Proposition 3.3 applied in the case where $\phi$ is the identity map on $M$.

THEOREM 3.5. Let sets of weights $S_{1}, S_{2}, \ldots, S_{k}$ be given such that $\operatorname{Hom}(M(\lambda), M(\mu))=0$ whenever $\lambda \in S_{i}$ and $\mu \in S_{j}$ with $i<j$. Suppose $M=M_{0} \supset M_{1} \supset M_{2} \supset \cdots$ is an RHWS with factors $F_{i}=$ $M_{i-1} / M_{i}$ of highest weight $\lambda_{i}$, and suppose that all $\lambda_{i} \in S_{1} \cup S_{2} \cup \cdots \cup$ $S_{k}$. Further suppose that for each $j=2,3, \ldots, k$, the set $\left\{i \mid \lambda_{i} \in S_{j}\right\}$ is a finite set. Then there is a unique filtration $0=N_{0} \subset N_{1} \subset \cdots \subset$ $N_{k}=M$ such that each $N_{j} / N_{j-1}$ has an RHWS with all factors having highest weights in $S_{j}$ for $j=1,2, \ldots, k$, with the RHWS being finite for $j=2,3, \ldots, k$. If $M^{\prime}$ is another module satisfying the hypotheses and $0=N_{0}^{\prime} \subset N_{1}^{\prime} \subset \cdots \subset N_{k}^{\prime}=M^{\prime}$ is its corresponding filtration, then for any $\phi \in \operatorname{Hom}\left(M, M^{\prime}\right)$ we have $\phi\left(N_{j}\right) \subset N_{j}^{\prime}$ for $j=0,1, \ldots, k$, so that $\phi$ induces maps $\phi_{j}: N_{j} / N_{j-1} \rightarrow N_{j}^{\prime} / N_{j-1}^{\prime}$ for $j=1,2, \ldots, k$.

Proof. This follows from Propositions 3.2 and 3.3 and Corollary 3.4, by induction on $k$.

We now apply Lemma 3.1 to the case of modules with HWS, obtaining similar filtrations, but without the extra finiteness assumption.

Proposition 3.6. Let sets of weights $S_{1}$ and $S_{2}$ be given such that $\operatorname{Hom}(M(\lambda), M(\mu))=0$ whenever $\lambda \in S_{1}$ and $\mu \in S_{2}$. Suppose $M$ has an HWS $0=M_{0} \subset M_{1} \subset M_{2} \subset \cdots$ where all factors have highest weights in $S_{1} \cup S_{2}$. Then there is a submodule $N$ of $M$ such that $N$ has an HWS, all of whose factors have highest weights in $S_{1}$, and such that $M / N$ has an HWS, all of whose factors have highest weights in $S_{2}$.

Proof. We first use induction on the number of factors to prove the result for modules with finite HWS. Thus, assume that for $M_{k}$ we have already found a submodule $N_{k}$ such that $N_{k}$ has a finite HWS whose factors have highest weights in $S_{1}$ and such that $M_{k} / N_{k}$ has a finite HWS whose factors have highest weights in $S_{2}$. Now, consider $M_{k+1}$. We have $0 \subset N_{k} \subset M_{k} \subset M_{k+1}$. If the factor $M_{k+1} / M_{k}$ has highest weight in $S_{2}$, then we simply set $N_{k+1}=N_{k}$. Otherwise, if the factor $M_{k+1} / M_{k}$ has highest weight in $S_{1}$, then repeated application of Lemma 3.1 allows us to switch the order of factors and produce a filtration $0 \subset N_{k} \subset N_{k+1} \subset M_{k+1}$, where $N_{k+1} / N_{k}$ has the same highest weight as $M_{k+1} / M_{k}$, and where $M_{k+1} / N_{k+1}$ has a finite HWS whose factors have highest weights in $S_{2}$. 
We thus have a sequence of submodules $0=N_{0} \subset N_{1} \subset N_{2} \subset \cdots$ of $M$, and by setting $N=\bigcup_{k \geq 0} N_{k}$, we see that $N$ has an HWS whose factors have highest weights in $S_{1}$. It remains to show that $M / N$ has the appropriate HWS. Consider $0=\left(M_{0}+N\right) / N \subset\left(M_{1}+N\right) / N \subset$ $\left(M_{2}+N\right) / N \subset \cdots$ as a filtration of $M / N$. Note that there may be some repetitions. We show that if repetitions are eliminated, then this is the desired HWS for $M / N$. Observe that each factor

$$
\left[\left(M_{k+1}+N\right) / N\right] /\left[\left(M_{k}+N\right) / N\right]
$$

is a homomorphic image of $M_{k+1} / M_{k}$, and hence it is either zero or a highest weight module of the same highest weight as $M_{k+1} / M_{k}$, which is therefore in $S_{1} \cup S_{2}$. On the other hand, that same factor module $\left[\left(M_{k+1}+N\right) / N\right] /\left[\left(M_{k}+N\right) / N\right]$ is also a homomorphic image of $M_{k+1}$ with $N_{k+1}$ contained in its kernel, and hence it is a homomorphic image of $M_{k+1} / N_{k+1}$, whose HWS has factors with highest weights in $S_{2}$. Thus, applying Proposition 2.9 and our hypothesis on $S_{1}$ and $S_{2}$, we see that the factor module

$$
\left[\left(M_{k+1}+N\right) / N\right] /\left[\left(M_{k}+N\right) / N\right]
$$

cannot have any composition factors of the form $L(\lambda)$ with $\lambda \in S_{1}$. We conclude that $\left[\left(M_{k+1}+N\right) / N\right] /\left[\left(M_{k}+N\right) / N\right]$ is either zero or a highest weight module with highest weight in $S_{2}$. Therefore, eliminating repetitions gives the desired conclusion on the HWS for $M / N$.

The following proposition, corollary, and theorem are analogous to Proposition 3.3, Corollary 3.4, and Theorem 3.5. Consequently, their proofs, which are similar to those dealing with RHWS, are left to the reader.

Proposition 3.7. Let sets of weights $S_{1}$ and $S_{2}$ be given such that $\operatorname{Hom}(M(\lambda), M(\mu))=0$ whenever $\lambda \in S_{1}$ and $\mu \in S_{2}$. Suppose that $M$ and $M^{\prime}$ each satisfy the hypotheses of Proposition 3.6, and further suppose that $N \subset M$ and $N^{\prime} \subset M^{\prime}$ are submodules satisfying the conclusion of Proposition 3.6. Then for any $\phi \in \operatorname{Hom}\left(M, M^{\prime}\right)$, we must have $\phi(N) \subset N^{\prime}$, so that $\phi$ induces maps $\phi_{1}: N \rightarrow N^{\prime}$ and $\phi_{2}: M / N \rightarrow M^{\prime} / N^{\prime}$.

Corollary 3.8. Let $S_{1}, S_{2}$, and $M$ satisfy the hypotheses of Proposition 3.6. Then the submodule $N$ given in the conclusion of Proposition 3.6 is unique. 
THEOREM 3.9. Let sets of weights $S_{1}, S_{2}, \ldots, S_{k}$ be given such that $\operatorname{Hom}(M(\lambda), M(\mu))=0$ whenever $\lambda \in S_{i}$ and $\mu \in S_{j}$ with $i<j$. Suppose $0=M_{0} \subset M_{1} \subset M_{2} \subset \cdots$ is an HWS for $M$ with factors $F_{i}=M_{i} / M_{i-1}$ of highest weight $\lambda_{i}$, and suppose that all $\lambda_{i} \in S_{1} \cup S_{2}$ $\cup \cdots \cup S_{k}$. Then there is a unique filtration $0=N_{0} \subset N_{1} \subset \cdots \subset N_{k}=$ $M$ such that each $N_{j} / N_{j-1}$ has an HWS with all factors having highest weights in $S_{j}$ for $j=1,2, \ldots, k$. If $M^{\prime}$ is another module satisfying the hypotheses and $0=N_{0}^{\prime} \subset N_{1}^{\prime} \subset \cdots \subset N_{k}^{\prime}=M^{\prime}$ is its corresponding filtration, then for any $\phi \in \operatorname{Hom}\left(M, M^{\prime}\right)$ we have $\phi\left(N_{j}\right) \subset N_{j}^{\prime}$ for $j=0,1, \ldots, k$, so that $\phi$ induces maps $\phi_{j}: N_{j} / N_{j-1} \rightarrow N_{j}^{\prime} / N_{j-1}^{\prime}$ for $j=1,2, \ldots, k$.

We conclude this section with a look at certain cases where modules do indeed have RHWS. The following lemma gives a criterion for distinguishing those modules with RHWS.

LEMMA 3.10. Let $M$ be a weight module. Then $M$ has an RHWS if and only if $M=\sum_{i=1}^{\infty} U\left(\mathfrak{n}^{-}\right) v_{i}$, where the $v_{i}$ are weight vectors of weights $\lambda_{i}$, respectively, and for any $\mu \in \mathfrak{h}^{*},\left\{i \mid \lambda_{i} \leq \mu\right\}$ is a finite set.

Proof. If $M$ has an RHWS, then such weight vectors $v_{i}$ exist by Definition 2.6. Conversely, suppose such $v_{i} \in M_{\lambda_{i}}$ exist. By rearranging the order of the $v_{i}$ if necessary, we may assume that $\lambda_{i}<\lambda_{j}$ implies $i<j$. Set $M_{i}=\sum_{j>i} U\left(\mathfrak{n}^{-}\right) v_{j}$ for each $i=0,1,2, \ldots$.

Comparing this with Definition 2.6, it remains to show that each $M_{i}$ is a submodule of $M$, and that each factor $F_{i}=M_{i-1} / M_{i}$ is either zero or a highest weight module of weight $\lambda_{i}$. To show that $M_{i}$ is a submodule, it suffices to show that for any $j=1,2,3, \ldots$, we have

$$
U(\mathfrak{g}) U\left(\mathfrak{n}^{-}\right) v_{j}=U(\mathfrak{g}) v_{j} \subset \sum_{k \geq j} U\left(\mathfrak{n}^{-}\right) v_{k}
$$

Since $U(\mathfrak{g})=U\left(\mathfrak{n}^{-}\right) U(\mathfrak{b})$ by the PBW Theorem, it suffices to show only that $U(\mathfrak{b}) v_{j} \subset \sum_{k \geq j} U\left(\mathfrak{n}^{-}\right) v_{k}$. But this is clear from the ordering of the weight vectors when we write $U(\mathfrak{b})=U\left(\mathfrak{n}^{+}\right) U(\mathfrak{h})$ by the PBW Theorem. Therefore each $M_{i}$ is a $\mathfrak{g}$-submodule of $M$. For the result on the factors, note that if $F_{i}=M_{i-1} / M_{i}$ is nonzero, then it is generated by the image of $v_{i}$ and that by the ordering of the weight vectors, $\mathfrak{n}^{+} \cdot v_{i} \subset M_{i}$, so that the image of $v_{i}$ is a highest weight vector and $F_{i}$ is a highest weight module of weight $\lambda_{i}$. 
Proposition 3.11. If $M$ has an RHWS and $N$ is a lowest weight module, then $M \otimes_{K} N$ has an $R H W S$.

Proof. By the above lemma, we may write $M=\sum_{i=1}^{\infty} U\left(\mathfrak{n}^{-}\right) v_{i}$, where the $v_{i}$ are weight vectors of weights $\lambda_{i}$, respectively, and for any $\mu \in \mathfrak{h}^{*},\left\{i \mid \lambda_{i} \leq \mu\right\}$ is a finite set. Choosing a $K$-basis $\left\{w_{1}, w_{2}\right.$, ... $\}$ of weight vectors for $N$, we see that $M \otimes_{K} N$ is generated over $U\left(\mathfrak{n}^{-}\right)$by the weight vectors $v_{i} \otimes w_{j}$ for $i, j=1,2,3, \ldots$. Since $N$ is a lowest weight module, it is clear that the finiteness condition of Lemma 3.10 is satisfied by the weights of the weight vectors $v_{i} \otimes w_{j}$. Therefore, we may apply Lemma 3.10 to conclude that $M \otimes_{K} N$ has an RHWS.

REMARK. This shows, in particular, that $M \otimes_{K} \Gamma(\mu)$ has an RHWS for any $M$ with an RHWS and for any $\mu \in \mathfrak{h}^{*}$.

4. Functors and an adjoint-like property. We begin by using the results of the previous section to define certain functors on modules with HWS or RHWS. We then prove an adjoint-like property of two of these functors.

Throughout this section we fix three sets of weights $S_{1}, S_{2}$, and $S_{3}$ such that $\mathfrak{h}^{*}=S_{1} \cup S_{2} \cup S_{3}$ and such that $\operatorname{Hom}(M(\lambda), M(\mu))=0$ whenever $\lambda \in S_{i}$ and $\mu \in S_{j}$ with $i<j$.

Definition 4.1. For any module $M$ with HWS, let $0=N_{0} \subset N_{1} \subset$ $N_{2} \subset N_{3}=M$ be the filtration given by Theorem 3.9 relative to the sets of weights $S_{1}, S_{2}$, and $S_{3}$. We denote the quotient $N_{2} / N_{1}$ by $M^{S_{2}}$. Also, if $\phi \in \operatorname{Hom}\left(M, M^{\prime}\right)$, where each of $M$ and $M^{\prime}$ have HWS, then we denote the induced map $\phi_{2}$ given in Theorem 3.9 by $\phi^{S_{2}}: M^{S_{2}} \rightarrow M^{\prime S_{2}}$. Thus the assignments $M \rightarrow M^{S_{2}}$ and $\phi \rightarrow \phi^{S_{2}}$ define a functor on the category of modules with HWS.

We define similarly a functor on the category of modules with RHWS with finitely many factors having highest weights in $S_{2} \cup S_{3}$, where we use again the notation $M \rightarrow M^{S_{2}}$ for the assignment of the "middle" subquotient given by Theorem 3.5 and $\phi \rightarrow \phi^{S_{2}}$ for the assignment of the map induced by $\phi$ on these subquotients, as given by Theorem 3.5 .

REMARK. Although we use the same notation for both functors, it will be clear from the context whether we are dealing with modules with HWS or modules with RHWS. In either case, $M^{S_{2}}$ denotes the subquotient whose factors in its HWS or RHWS have highest weights 
in $S_{2}$, and $\phi^{S_{2}}$ simply denotes the map induced by $\phi$ on these subquotients, so this should not cause any confusion.

In the following definition and lemma, we will be working with resolutions of modules by modules with VS. It should be noted that for any module with HWS, one can always construct such a resolution. To see this, first recall the standard resolution

$$
\cdots \rightarrow U(\mathfrak{g}) \otimes_{U(\mathfrak{b})} \bigwedge^{q}(\mathfrak{g} / \mathfrak{b}) \rightarrow \cdots \rightarrow U(\mathfrak{g}) \otimes_{U(\mathfrak{b})} \bigwedge^{0}(\mathfrak{g} / \mathfrak{b}) \rightarrow K \rightarrow 0
$$

of $K$ constructed and shown to be exact in [1, Theorem II.9.1]. By tensoring this resolution with $M$ over $K$, we obtain the desired resolution of $M$, because for each $q,\left(U(\mathfrak{g}) \otimes_{U(\mathfrak{b})} \bigwedge^{q}(\mathfrak{g} / \mathfrak{b})\right) \otimes_{K} M \cong$ $U(\mathfrak{g}) \otimes_{U(\mathfrak{b})}\left(\bigwedge^{q}(\mathfrak{g} / \mathfrak{b}) \otimes_{K} M\right)$ by [3, Proposition 1.7], and this latter module is easily seen to have a VS by choosing a $K$-basis of $\Lambda^{q}(\mathfrak{g} / \mathfrak{b}) \otimes_{K} M$ consisting of weight vectors.

Definition 4.2. Let $M$ be a module with HWS. Then for each $q=0,1,2, \ldots$, we define $W_{q}^{S_{2}} M$ to be the $q$ th homology of the complex

$$
\cdots \rightarrow M_{2}^{S_{2}} \rightarrow M_{1}^{S_{2}} \rightarrow M_{0}^{S_{2}} \rightarrow 0
$$

where

$$
\cdots \rightarrow M_{2} \rightarrow M_{1} \rightarrow M_{0} \rightarrow M \rightarrow 0
$$

is any resolution of $M$ by modules with VS. Furthermore if $M^{\prime}$ is another module with HWS, and if $\phi \in \operatorname{Hom}\left(M, M^{\prime}\right)$, then, using the standard resolution of $K$ to obtain resolutions of both $M$ and $M^{\prime}$, we see that $\phi$ induces a morphism of complexes, and we denote by $W_{q}^{S_{2}} \phi: W_{q}^{S_{2}} M \rightarrow W_{q}^{S_{2}} M^{\prime}$ the map induced on homology.

REMARK. In the above definition, it is not clear whether the module $W_{q}^{S_{2}} M$ depends on the choice of resolution. The functors $M \rightarrow$ $W_{q}^{S_{2}} M$ form a sequence of locally derived functors introduced in [6] and used extensively in both [6] and [7]. A better approach than that of the above definition is to use local projective resolutions, where the projectivity is used to guarantee independence from the choice of resolution. It was shown, however, in [6, Theorem 6.2] that resolutions by modules with VS may be used to obtain the same results. Since for our purposes we will not need to make extensive use of local projective resolutions, we choose to use the above definition here. Also, we will almost exclusively work with the functor $W_{0}^{S_{2}}$. This functor is right exact. 
LEMMA 4.3. Let $M$ be a module with HWS. If all the composition factors of $M$ have highest weights in $S_{2} \cup S_{3}$, then $M$ has a resolution by modules with VS, all of whose Verma factors have highest weights in $S_{2} \cup S_{3}$. Furthermore, if all the composition factors of $M$ have highest weights in $S_{3}$, then the modules with VS may also be chosen to have all factors with highest weights in $S_{3}$.

Proof. For the first statement, it suffices to show that there is a short exact sequence

$$
0 \rightarrow N \rightarrow V \rightarrow M \rightarrow 0
$$

in which $V$ has a VS whose factors all have highest weights in $S_{2} \cup$ $S_{3}$, and in which $N$ satisfies the same hypotheses as $M$, since we may then repeat the argument on $N$ and splice together the resulting short exact sequences. We begin with an epimorphism $\phi: X \rightarrow M$ in which $X$ has a VS. Such an epimorphism exists, since we may choose, for example, the tail end of any resolution of $M$ by modules with VS. Let $0=N_{0} \subset N_{1} \subset N_{2} \subset N_{3}=M$ be the filtration of $M$ given by Theorem 3.9, and let $0=Y_{0} \subset Y_{1} \subset Y_{2} \subset Y_{3}=X$ be the corresponding filtration of $X$ given by Theorem 3.9. Our hypothesis on $M$ implies that $N_{1}=0$. Also, it was shown in [6, Corollary 4.4] that the factors of the filtration of $X$ have VS, not just HWS, since nothing is lost when the order of Verma factors is reversed. Now Theorem 3.9 shows that $\phi\left(Y_{1}\right) \subset N_{1}=0$, so that $Y_{1} \subset \operatorname{Ker} \phi$. Hence $\phi$ induces an epimorphism $\psi: X / Y_{1} \rightarrow M$. Setting $V=X / Y_{1}$ and $N=\operatorname{Ker} \psi$ gives us the desired short exact sequence, with the appropriate Verma factors. The condition on the composition factors of $N$ follows from Proposition 2.9, since that proposition may clearly be applied to obtain the result for $V$ and $N \subset V$.

For the second statement, the proof is similar, and is left to the reader.

LEMMA 4.4. Let $M$ be a module with HWS. If all the factors of the $H W S$ have highest weights in $S_{2}$, then there is a natural epimorphism $\pi: W_{0}^{S_{2}} M \rightarrow M$ in which all the composition factors of $\operatorname{Ker} \pi$ have highest weights in $S_{3}$.

Proof. Our hypothesis on the HWS for $M$ means that we can identify $M^{S_{2}}$ with $M$. From Lemma 4.3, there exists an epimorphism $\phi: V \rightarrow M$ where $V$ has a VS whose Verma factors have highest weights in $S_{2} \cup S_{3}$. We can then identify $V^{S_{2}}$ with a submodule of $V$. 
Consider the filtration $0=\phi(0) \subset \phi\left(V^{S_{2}}\right) \subset \phi(V)=M$. Since $V^{S_{2}}$ has a VS whose factors all have highest weights in $S_{2}$, its homomorphic image $\phi\left(V^{S_{2}}\right)$ has an HWS whose factors all have highest weights in $S_{2}$. Similarly, since $V / V^{S_{2}}$ has a VS whose factors all have highest weights in $S_{3}$, its homomorphic image $\phi(V) / \phi\left(V^{S_{2}}\right)$ has an HWS whose factors all have highest weights in $S_{3}$. But the same is true of the HWS of the factors of the filtration $0=0 \subset M^{S_{2}}=M=M$. By the uniqueness statement of Theorem 3.9 applied to the sets of weights $S_{2}$ and $S_{3}$, we must have $\phi\left(V^{S_{2}}\right)=M^{S_{2}}=M$. Set $M_{0}=V^{S_{2}}$. We now have an epimorphism $\psi: M_{0} \rightarrow M$. Letting $N=\operatorname{Ker} \psi$, we have by Proposition 2.9 that all composition factors of $N$ have highest weights in $S_{2} \cup S_{3}$. Applying Lemma 4.3 to $N$, we obtain a resolution

$$
\cdots \rightarrow M_{2} \rightarrow M_{1} \rightarrow N \rightarrow 0
$$

of $N$ by modules with VS whose Verma factors all have highest weights in $S_{2} \cup S_{3}$. Splicing this sequence with the short exact sequence

$$
0 \rightarrow N \rightarrow M_{0} \rightarrow M \rightarrow 0
$$

we obtain a resolution

$$
\cdots \rightarrow M_{2} \rightarrow M_{1} \stackrel{\xi}{\rightarrow} M_{0} \rightarrow M \rightarrow 0
$$

of $M$ by modules with VS whose Verma factors all have highest weights in $S_{2} \cup S_{3}$, in which all the Verma factors of $M_{0}$ have highest weights in $S_{2}$. Note that we may identify $M_{0}^{S_{2}}$ with $M_{0}$ and that we may identify $M_{1}^{S_{2}}$ with a submodule of $M_{1}$. Since the resolution is exact, we have $M \cong M_{0} / \xi\left(M_{1}\right)$. Also, using Definition 4.2, we have $W_{0}^{S_{2}} M \cong M_{0} / \xi\left(M_{1}^{S_{2}}\right)$. Since $\xi\left(M_{1}^{S_{2}}\right) \subset \xi\left(M_{1}\right)$ we see that there is an epimorphism $\pi: W_{0}^{S_{2}} M \rightarrow M$ with $\operatorname{Ker} \pi \cong \xi\left(M_{1}\right) / \xi\left(M_{1}^{S_{2}}\right)$. Note that $\operatorname{Ker} \pi$ is a homomorphic image of $M_{1} / M_{1}^{S_{2}}$, so that by Proposition 2.9, all composition factors of $\operatorname{Ker} \pi$ have highest weights in $S_{3}$.

Finally observe that all of the above constructions, including those used in the proof of Lemma 4.3, depend only on choices of resolutions by modules with VS. Since we may always work with resolutions which are constructed from the standard resolution as in the discussion preceding Definition 4.2, the naturality follows.

We are now ready to prove an adjoint-like property of two of our functors. 
THEOREM 4.5. Suppose $M$ is a module with HWS such that all composition factors of $M$ have highest weights in $S_{2} \cup S_{3}$, and suppose that $V$ is a module with RHWS such that all the factors of the RHWS for $V$ have highest weights in $S_{1} \cup S_{2}$, finitely many being in $S_{2}$. Then there is a natural isomorphism $\operatorname{Hom}\left(V, W_{0}^{S_{2}} M\right) \cong \operatorname{Hom}\left(V^{S_{2}}, M\right)$.

Proof. First observe that for any such $V$, there is a short exact sequence

$$
0 \rightarrow X \rightarrow V \rightarrow V^{S_{2}} \rightarrow 0
$$

in which $X$ has a RHWS whose factors all have highest weights in $S_{1}$. Thus $\operatorname{Hom}\left(X, W_{0}^{S_{2}} M\right)=0$, and we have a natural isomorphism $\operatorname{Hom}\left(V, W_{0}^{S_{2}} M\right) \cong \operatorname{Hom}\left(V^{S_{2}}, W_{0}^{S_{2}} M\right)$.

On the other hand, for any such $M$, there is a short exact sequence

$$
0 \rightarrow M^{S_{2}} \rightarrow M \rightarrow Y \rightarrow 0
$$

in which $Y$ has a HWS, all of whose factors have highest weights in $S_{3}$. By Lemma 4.3 and Definition 4.2, we have $W_{q}^{S_{2}} Y=0$ for all $q$, and by the long exact sequence for locally derived functors [7, Theorem 3.7] we have $W_{0}^{S_{2}} M \cong W_{0}^{S_{2}}\left(M^{S_{2}}\right)$. The functoriality of $W_{0}^{S_{2}}$ shows that this isomorphism is natural. Thus we also have a natural isomorphism $\operatorname{Hom}\left(V^{S_{2}}, W_{0}^{S_{2}} M\right) \cong \operatorname{Hom}\left(V^{S_{2}}, W_{0}^{S_{2}}\left(M^{S_{2}}\right)\right)$.

Now, from Lemma 4.4, we have a natural epimorphism

$$
\pi: W_{0}^{S_{2}}\left(M^{S_{2}}\right) \rightarrow M^{S_{2}}
$$

in which $\operatorname{Ker} \pi$ has all its composition factors of highest weight in $S_{3}$. Proposition 2.9 shows that $\operatorname{Ext}^{n}\left(V^{S_{2}}, \operatorname{Ker} \pi\right)=0$ for all $n$, so that in particular we have a natural isomorphism $\operatorname{Hom}\left(V^{S_{2}}, W_{0}^{S_{2}}\left(M^{S_{2}}\right)\right) \cong$ $\operatorname{Hom}\left(V^{S_{2}}, M^{S_{2}}\right)$. Finally, Proposition 2.9 shows that $\operatorname{Hom}\left(V^{S_{2}}, Y\right)=$ 0 , so that we obtain a natural isomorphism

$$
\operatorname{Hom}\left(V^{S_{2}}, M^{S_{2}}\right) \cong \operatorname{Hom}\left(V^{S_{2}}, M\right) \text {. }
$$

The result now follows by composing the above natural isomorphism.

5. Translation functors. We will now use the functors defined in the previous section to define the translation functors. The basic idea is to "translate" Verma modules from one $W$-orbit to another (where orbits refer to the dot action of $W$ ). Although we could consider translation in more generality, as in [7], for our present purposes we 
need only consider translation back and forth between the $W$-orbit of a weight on one wall of the dominant Weyl chamber and the $W$-orbit of a weight in the dominant chamber which is "close" to that wall.

Throughout this section, we fix a choice of $i \in\{1,2, \ldots, l\}$, we fix $\lambda \in P^{+}$such that $\lambda\left(h_{i}\right)=0$, and fix $\eta \in P^{+}$such that $\eta\left(h_{i}\right)=$ 1 and $\eta\left(h_{j}\right)=0$ for all $j \neq i$. Set $\alpha=\alpha_{i}$ and set $s=s_{i}$, so that $\alpha$ is a simple root and $s$ is the corresponding simple reflection in $W$. Observe that for the extreme weight $s \eta$ of $L(\eta)$, we have $s \eta=\eta-\alpha$. Let $\lambda_{\alpha}=\lambda+s \eta=\lambda+\eta-\alpha$. Note that $\lambda_{\alpha}\left(h_{i}\right)=-1$, so that $s \cdot \lambda_{\alpha}=\lambda_{\alpha}$, and further note that $\lambda_{\alpha}\left(h_{j}\right) \geq 0$ for all $j \neq i$. Thus $\lambda_{\alpha}$ is on the $\alpha$-wall of the dominant Weyl chamber with respect to the dot action of $W$ on $\mathfrak{h}^{*}$, and $\lambda$ is "close" to that wall. This restriction on $\lambda$ may seem artificial at first, but the reason for this is that when we begin to apply translation functors, certain $W$-orbits become interchanged. With our choice of $\lambda$ and $\lambda_{\alpha}$, only two of these orbits are interchanged, and we may sort these two out by the representation theory of $\mathrm{sl}(2, K)$, as we do in Proposition 5.1(iv) below.

We now consider certain sets which are unions of $W$-orbits, and show that they have many properties which will allow us to apply the results of the previous section.

Proposition 5.1. Let $S_{1}^{\prime}=\left\{\nu \in \mathfrak{h}^{*} \mid w \cdot \nu \not \leq \lambda\right.$ for some $\left.w \in W\right\}$, $S_{2}^{\prime}=W \cdot \lambda$, and $S_{3}^{\prime}=\left\{\nu \in \mathfrak{h}^{*} \mid w \cdot \nu<\lambda\right.$ for all $\left.w \in W\right\}$. Also, let $S_{1}^{\prime \prime}=\left\{\nu \in \mathfrak{h}^{*} \mid w \cdot \nu \not \leq \lambda+\eta\right.$ for some $\left.w \in W\right\}, S_{2}^{\prime \prime}=W \cdot \lambda_{\alpha}$, and $S_{3}^{\prime \prime}=\left\{\nu \in \mathfrak{h}^{*} \mid \nu \notin S_{2}^{\prime \prime}\right.$ and $w \cdot \nu \leq \lambda+\eta$ for all $\left.w \in W\right\}$. Then all of the following hold.

(i) $\mathfrak{h}^{*}$ is the disjoint union of $S_{1}^{\prime}, S_{2}^{\prime}$, and $S_{3}^{\prime}$.

(ii) $\mathfrak{h}^{*}$ is the disjoint union of $S_{1}^{\prime \prime}, S_{2}^{\prime \prime}$, and $S_{3}^{\prime \prime}$.

(iii) $\operatorname{Hom}\left(M\left(\nu_{i}\right), M\left(\nu_{j}\right)\right)=0$ whenever $\nu_{i} \in S_{i}^{\prime}$ and $\nu_{j} \in S_{j}^{\prime}$ with $i<j$.

(iv) $\operatorname{Hom}\left(M\left(\nu_{i}\right), M\left(\nu_{j}\right)\right)=0$ whenever $\nu_{i} \in S_{i}^{\prime \prime}$ and $\nu_{j} \in S_{j}^{\prime \prime}$ with $i<j$.

(v) For any $\nu \in S_{3}^{\prime}$ and any $\chi \in \Pi(L(\eta)), \nu+\chi \in S_{3}^{\prime \prime}$.

(vi) For any $\nu \in S_{2}^{\prime}$ and any $\chi \in \Pi(L(\eta)), \nu+\chi \in S_{2}^{\prime \prime} \cup S_{3}^{\prime \prime}$.

(vii) For any $\nu \in S_{1}^{\prime \prime}$ and any $\chi \in \Pi(\Gamma(-\eta)), \nu+\chi \in S_{1}^{\prime}$.

(viii) For any $\nu \in S_{2}^{\prime \prime}$ and any $\chi \in \Pi(\Gamma(-\eta)), \nu+\chi \in S_{1}^{\prime} \cup S_{2}^{\prime}$.

Proof. Statements (i) and (ii) are clear. Statements (iii) and (iv) follow from [6, Corollary 5.4] and the fact that 


$$
\operatorname{Hom}\left(M\left(\lambda_{\alpha}\right), M(\lambda+\eta)\right)=0
$$

by the representation theory of $\operatorname{sl}(2, K)$. Statements (v) and (vi) follow from the fact that for any $w \in W, w \cdot(\nu+\chi)=w \cdot \nu+w \chi$ and we always have $w \chi \in \Pi(L(\eta))$ so that $w \chi \leq \eta$.

To prove (vii), let $\nu \in S_{1}^{\prime \prime}$ and let $\chi \in \Pi(\Gamma(-\eta))$. Choosing $w \in W$ so that $w \cdot \nu \not \lambda \lambda+\eta$, we must also have $w \chi \geq-\eta$, so that $w \cdot(\nu+\chi)=$ $w \cdot \nu+w \chi \not \lambda$, and therefore $\nu+\chi \in S_{1}^{\prime}$.

To prove (viii), let $\nu \in S_{2}^{\prime \prime}$ and $\chi \in \Pi(\Gamma(-\eta))$. Then $\nu=w \cdot \lambda_{\alpha}$ for some $w \in W$, and we have $w^{-1} \cdot(\nu+\chi)=w^{-1} \cdot \nu+w^{-1} \chi=$ $\lambda_{\alpha}+w^{-1} \chi=(\lambda+\eta-\alpha)+w^{-1} \chi=\lambda+\left(\eta+w^{-1} \chi\right)-\alpha$. Now $w^{-1} \chi \geq-\eta$, so that we may write $\eta+w^{-1} \chi=\sum_{i=1}^{l} n_{j} \alpha_{j}$ with all $n_{j} \in \mathbb{Z}_{+}$. If any $n_{j} \neq 0$ with $j \neq i$, then the above computation shows that $w^{-1} \cdot(\nu+\chi) \not \lambda$, and we then would have $\nu+\chi \in S_{1}^{\prime}$. Thus, we may assume $\eta+w^{-1} \chi=n_{i} \alpha$. But then $-\eta+n_{i} \alpha=w^{-1} \chi \in \Pi(\Gamma(-\eta))$, and since $\eta\left(h_{i}\right)=1$, there are only two possibilities for $n_{i}$, namely 0 and 1 . In case $n_{i}=1$, we have $w^{-1} \cdot(\nu+\chi)=\lambda+\alpha-\alpha=\lambda$, and therefore $\nu+\chi \in S_{2}^{\prime}$. Thus, we may assume that $n_{i}=0$. This means that $w^{-1} \chi=-\eta$, so that $s w^{-1} \chi=-s \eta=-(\eta-\alpha)=-\eta+\alpha$. But we also have $\left(s w^{-1}\right) \cdot \nu=s \cdot\left(w^{-1} \cdot \nu\right)=\lambda_{\alpha}$, so that $\left(s w^{-1}\right) \cdot(\nu+\chi)=$ $\left(s w^{-1}\right) \cdot \nu+\left(s w^{-1}\right) \chi=\lambda_{\alpha}-\eta+\alpha=\lambda$, and therefore $\nu+\chi \in S_{2}^{\prime}$.

Definition 5.2. Let $S_{1}^{\prime}, S_{2}^{\prime}, S_{3}^{\prime}, S_{1}^{\prime \prime}, S_{2}^{\prime \prime}$, and $S_{3}^{\prime \prime}$ be as in Proposition 5.1. We define the translation functors $T_{\lambda}^{\lambda_{\alpha}}$ and $T_{\lambda_{\alpha}}^{\lambda}$ by $T_{\lambda}^{\lambda_{\alpha}} M=$ $W_{0}^{S_{2}^{\prime \prime}}\left(W_{0}^{S_{2}^{\prime}} M \otimes_{K} L(\eta)\right)$ and $T_{\lambda_{\alpha}}^{\lambda} M=\left(M^{S_{2}^{\prime \prime}} \otimes_{K} \Gamma(-\eta)\right)^{S_{2}^{\prime}}$. The functor $T_{\lambda}^{\lambda_{\alpha}}$ is a functor from the category $C(\lambda)$ to the category $C\left(\lambda_{\alpha}\right)$, but the functor $T_{\lambda_{\alpha}}^{\lambda}$ is a functor from a subcategory of $C\left(\lambda_{\alpha}\right)$ to the category $C(\lambda)$, as it is only defined on those $M$ in $C\left(\lambda_{\alpha}\right)$ with an RHWS which has only finitely many factors of highest weight in $S_{2}^{\prime \prime} \cup S_{3}^{\prime \prime}$.

REMARK. In the sequel, we will make use of the fact that $T_{\lambda}^{\lambda_{\alpha}}$ is a composition of right exact functors, and hence is itself right exact.

Theorem 5.3. Let $S_{1}^{\prime}, S_{2}^{\prime}, S_{3}^{\prime}, S_{1}^{\prime \prime}, S_{2}^{\prime \prime}$, and $S_{3}^{\prime \prime}$ be as in Proposition 5.1. If $M \in \mathrm{Ob} C\left(\lambda_{\alpha}\right)$ has a finite $H W S$ with all factors of highest weight in $S_{1}^{\prime \prime} \cup S_{2}^{\prime \prime}$, and if $N \in \mathrm{Ob} C(\lambda)$ has all its composition factors of highest weight in $S_{2}^{\prime} \cup S_{3}^{\prime}$, then there is a natural isomorphism $\operatorname{Hom}\left(T_{\lambda_{\alpha}}^{\lambda} M, N\right) \cong \operatorname{Hom}\left(M, T_{\lambda}^{\lambda^{\alpha}} N\right)$. 
Proof. By Definition 5.2,

$$
\operatorname{Hom}\left(T_{\lambda_{\alpha}}^{\lambda} M, N\right)=\operatorname{Hom}\left(\left(M^{S_{2}^{\prime \prime}} \otimes_{K} \Gamma(-\eta)\right)^{S_{2}^{\prime}}, N\right) .
$$

Using the finite HWS for $M^{S_{2}^{\prime \prime}}$, we see by Propositions 3.11 and 5.1(viii) that $M^{S_{2}^{\prime \prime}} \otimes_{K} \Gamma(-\eta)$ has an RHWS with all its factors having highest weights in $S_{1}^{\prime} \cup S_{2}^{\prime}$, and only finitely many are in $S_{2}^{\prime}$. Thus, we may apply Theorem 4.5 to obtain $\operatorname{Hom}\left(\left(M^{S_{2}^{\prime \prime}} \otimes_{K} \Gamma(-\eta)\right)^{S_{2}^{\prime}}, N\right) \cong$ $\operatorname{Hom}\left(M^{S_{2}^{\prime \prime}} \otimes_{K} \Gamma(-\eta), W_{0}^{S_{2}^{\prime}} N\right)$. Now, by [7, Lemma 7.7],

$$
\operatorname{Hom}\left(M^{S_{2}^{\prime \prime}} \otimes_{K} \Gamma(-\eta), W_{0}^{S_{2}^{\prime}} N\right) \cong \operatorname{Hom}\left(M^{S_{2}^{\prime \prime}}, W_{0}^{S_{2}^{\prime}} \otimes_{K} L(\eta)\right) .
$$

From Propositions 2.9 and Proposition 5.1(iii), we have that all the composition factors of $W_{0}^{S_{2}^{\prime}} N$ have highest weights in $S_{2}^{\prime} \cup S_{3}^{\prime}$, and hence, by Proposition 5.1(v) and (vi), all the composition factors of $W_{0}^{S_{2}^{\prime}} N \otimes_{K} L(\eta)$ have highest weights in $S_{2}^{\prime \prime} \cup S_{3}^{\prime \prime}$. Thus, we may again apply Theorem 4.5 to obtain

$$
\begin{aligned}
\operatorname{Hom}\left(M_{2}^{S_{2}^{\prime \prime}}, W_{0}^{S_{2}^{\prime}} N \otimes_{K} L(\eta)\right) & \cong \operatorname{Hom}\left(M, W_{0}^{S_{2}^{\prime \prime}}\left(W_{0}^{S_{2}^{\prime}} N \otimes_{K} L(\eta)\right)\right) \\
& =\operatorname{Hom}\left(M, T_{\lambda}^{\lambda} N\right) .
\end{aligned}
$$

We now extend this adjoint-like property to the Ext functors. To do this, we first need to study what happens to projective modules under translation. As we will see in the next two lemmas, the result is a projective module, but in the wrong category, and this is why we make the following definition.

Definition 5.4. The category $C(\lambda)_{\text {nice }}$ is defined to be the full subcategory of $C(\lambda)$ consisting of those modules whose composition factors all have highest weights in $S_{2}^{\prime} \cup S_{3}^{\prime}$, where $S_{2}^{\prime}$ and $S_{3}^{\prime}$ are as in Proposition 5.1.

We continue to use the notation of Proposition 5.1 in the following two lemmas and theorem.

LEMMA 5.5. Let $V \in \mathrm{Ob} C(\lambda)$ have a finite VS whose factors are all of the form $M(\nu)$ with $\nu \in S_{1}^{\prime} \cup S_{2}^{\prime}$, and let $M \in \mathrm{Ob} C(\lambda)_{\text {nice. }}$. Then there is a natural isomorphism $\operatorname{Ext}_{C(\lambda)}^{n}(V, M) \cong \operatorname{Ext}_{C(\lambda)}^{n}\left(V_{\text {nice }}\left(S_{2}^{\prime}, M\right)\right.$.

Proof. Let

$$
\cdots \rightarrow P_{n} \rightarrow \cdots \rightarrow P_{0} \rightarrow V \rightarrow 0
$$


be a projective resolution of $V$ in $C(\lambda)$ as in Proposition 2.8. Observe that the VS for each $P_{n}$ satisfies the same hypotheses as the VS for $V$, by Propositions 2.7 and 2.9. Applying [6, Theorem 4.8] gives the exact resolution

$$
\cdots \rightarrow P_{n}^{S_{2}^{\prime}} \rightarrow \cdots \rightarrow P_{0}^{S_{2}^{\prime}} \rightarrow V^{S_{2}^{\prime}} \rightarrow 0
$$

of $V^{S_{2}^{\prime}}$ in $C(\lambda)_{\text {nice }}$. We now note that each $P_{n}^{S_{2}^{\prime}}$ is projective in $C(\lambda)_{\text {nice }}$, since applying the functor $\operatorname{Hom}\left(P_{n}^{S_{2}^{\prime}},-\right)$ to a short sequence of modules in $C(\lambda)_{\text {nice }}$ is naturally equivalent to applying the exact functor $\operatorname{Hom}\left(P_{n},-\right)$ to the same short exact sequence, by [7, Lemma 7.3]. Thus $(*)$ is an exact projective resolution of $V^{S_{2}^{\prime}}$ in $C(\lambda)_{\text {nice }}$. Thus $\operatorname{Ext}_{C(\lambda)_{\text {nice }}}^{n}\left(V^{S_{2}^{\prime}}, M\right)$ is the $n$th cohomology of the complex

$$
0 \rightarrow \operatorname{Hom}\left(P_{0}^{S_{2}^{\prime}}, M\right) \rightarrow \cdots \rightarrow \operatorname{Hom}\left(P_{n}^{S_{2}^{\prime}}, M\right) \rightarrow \cdots,
$$

which, again using [7, Lemma 7.3], is naturally isomorphic to the complex

$$
0 \rightarrow \operatorname{Hom}\left(P_{0}, M\right) \rightarrow \cdots \rightarrow \operatorname{Hom}\left(P_{n}, M\right) \rightarrow \cdots,
$$

whose $n$th cohomology is $\operatorname{Ext}_{C(\lambda)}^{n}(V, M)$.

LEMMA 5.6. If $\nu \in S_{1}^{\prime \prime} \cup S_{2}^{\prime \prime}$, then $T_{\lambda_{\alpha}}^{\lambda} I_{\alpha}^{\lambda_{\alpha}}(\nu)$ is projective in $C(\lambda)_{\text {nice }}$.

Proof. We need only show that the functor $\operatorname{Hom}\left(T_{\lambda_{\alpha}}^{\lambda} I^{\lambda_{\alpha}}(\nu),-\right)$ is right exact when applied to sequences of modules in $C(\lambda)_{\text {nice }}$. Observe that the hypothesis on $\nu$ also holds for any $\chi$ such that $M(\chi)$ is a Verma factor of $I^{\lambda}(\nu)$, by Propositions 2.7 and 2.9, so that we have a natural isomorphism $\operatorname{Hom}\left(T_{\lambda_{\alpha}}^{\lambda} I_{\alpha}^{\lambda_{\alpha}}(\nu), M\right) \cong \operatorname{Hom}\left(I^{\lambda_{\alpha}}(\nu), T_{\lambda}^{\lambda_{\alpha}} M\right)$ for any $M \in \mathrm{Ob} C(\lambda)_{\text {nice }}$, by Theorem 5.3. Thus, given an exact sequence

$$
M^{\prime} \rightarrow M \rightarrow M^{\prime \prime} \rightarrow 0
$$

in $C(\lambda)_{\text {nice }}$, we may use the right exactness of $T_{\lambda}^{\lambda_{\alpha}}$ and the projectivity of $I^{\lambda_{\alpha}}(\nu)$ to obtain the exactness of

$$
\begin{aligned}
\operatorname{Hom}\left(I^{\lambda_{\alpha}}(\nu), T_{\lambda}^{\lambda_{\alpha}} M^{\prime}\right) & \rightarrow \operatorname{Hom}\left(I^{\lambda_{\alpha}}(\nu), T_{\lambda}^{\lambda_{\alpha}} M\right) \\
& \rightarrow \operatorname{Hom}\left(I^{\lambda_{\alpha}}(\nu), T_{\lambda}^{\lambda_{\alpha}} M^{\prime \prime}\right) \rightarrow 0,
\end{aligned}
$$

and applying the natural isomorphism noted above gives the exactness of

$$
\begin{aligned}
\operatorname{Hom}\left(T_{\lambda_{\alpha}}^{\lambda} I^{\lambda_{\alpha}}(\nu), M^{\prime}\right) & \rightarrow \operatorname{Hom}\left(T_{\lambda_{\alpha}^{\lambda}}^{I^{2}} I_{\alpha}(\nu), M\right) \\
& \rightarrow \operatorname{Hom}\left(T_{\lambda_{\alpha}}^{\lambda^{\lambda}} I^{\lambda_{\alpha}}(\nu), M^{\prime \prime}\right) \rightarrow 0
\end{aligned}
$$


THEOREM 5.7. Let $V \in \mathrm{Ob} C\left(\lambda_{\alpha}\right)$ have a finite $V S$ whose factors are all of the form $M(\nu)$ with $\nu \in S_{1}^{\prime \prime} \cup S_{2}^{\prime \prime}$, and let $M \in \mathrm{Ob} C(\lambda)_{\text {nice }}$. Then we have natural isomorphisms $\operatorname{Ext}_{C\left(\lambda_{\alpha}\right)}^{n}\left(V, T_{\lambda}^{\lambda_{\alpha}} M\right) \cong \operatorname{Ext}_{C(\lambda)}^{n}\left(T_{\lambda_{\alpha}}^{\lambda} V, M\right)$ for all $n=0,1, \ldots$.

Proof. By Propositions 2.7, 2.8, and 2.9, $V$ has a projective resolution

$$
\cdots \rightarrow P_{n} \rightarrow \cdots \rightarrow P_{0} \rightarrow V \rightarrow 0
$$

in $C\left(\lambda_{\alpha}\right)$ in which each $P_{n}$ is a finite direct sum of indecomposable projectives satisfying the hypotheses of Lemma 5.6, and in which all images and kernels have a VS satisfying the same hypotheses as the VS for $V$. Thus, using [6, Theorem 4.8] twice and the exactness of the functor $-\otimes_{K} \Gamma(-\eta)$ once, we see that

$$
\cdots \rightarrow T_{\lambda_{\alpha}}^{\lambda} P_{n} \rightarrow \cdots \rightarrow T_{\lambda_{\alpha}}^{\lambda} P_{0} \rightarrow T_{\lambda_{\alpha}}^{\lambda} V \rightarrow 0
$$

is an exact resolution of $T_{\lambda}^{\lambda} V$ in $C(\lambda)_{\text {nice }}$. By lemma 5.6, it is in fact a projective resolution of $T_{\lambda_{\alpha}}^{\lambda} V$ in $C(\lambda)_{\text {nice }}$. Thus, $\operatorname{Ext}_{C(\lambda)_{\text {nice }}}^{n}\left(T_{\lambda_{\alpha}}^{\lambda} V, M\right)$ is the $n$th cohomology of the complex

$$
0 \rightarrow \operatorname{Hom}\left(T_{\lambda_{\alpha}}^{\lambda} P_{0}, M\right) \rightarrow \cdots \rightarrow \operatorname{Hom}\left(T_{\lambda_{\alpha}}^{\lambda} P_{n}, M\right) \rightarrow \cdots .
$$

By Theorem 5.3, this complex is naturally isomorphic to the complex

$$
0 \rightarrow \operatorname{Hom}\left(P_{0}, T_{\lambda}^{\lambda^{\alpha}} M\right) \rightarrow \cdots \rightarrow \operatorname{Hom}\left(P_{n}, T_{\lambda}^{\lambda^{\alpha}} M\right) \rightarrow \cdots,
$$

whose $n$th cohomology is $\operatorname{Ext}_{C\left(\lambda_{\alpha}\right)}^{n}\left(V, T_{\lambda}^{\lambda_{\alpha}} M\right)$. We have shown that there is a natural isomorphism

$$
\operatorname{Ext}_{C(\lambda)_{\text {nce }}}^{n}\left(T_{\lambda_{\alpha}}^{\lambda} V, M\right) \cong \operatorname{Ext}_{C\left(\lambda_{\alpha}\right)}^{n}\left(V, T_{\lambda}^{\lambda_{\alpha}} M\right),
$$

and the result now follows from Lemma 5.5

We wish to compose translation functors to obtain the operators of coherent continuation. In order to do this, for technical reasons we cannot simply translate back to the same orbit. We now fix $\mu=\lambda_{\alpha}+\eta$ for the remainder of this section. The idea of coherent continuation is to translate from the orbit of $\lambda$ to the orbit of $\lambda_{\alpha}$ on the $\alpha$-wall, and then translate from the orbit of $\lambda_{\alpha}$ to the orbit of $\mu$.

Proposition 5.8. Let $S_{1}^{\prime}=\left\{\nu \in \mathfrak{h}^{*} \mid w \cdot \nu \not \leq \lambda_{\alpha}\right.$ for some $\left.w \in W\right\}$, $S_{2}^{\prime}=W \cdot \lambda_{\alpha}$, and $S_{3}^{\prime}=\left\{\nu \in \mathfrak{h}^{*} \mid w \cdot \nu<\lambda_{\alpha}\right.$ for all $\left.w \in W\right\}$. Also, let $S_{1}^{\prime \prime}=\left\{\nu \in \mathfrak{h}^{*} \mid w \cdot \nu \not \leq \mu\right.$ for some $\left.w \in W\right\}, S_{2}^{\prime \prime}=W \cdot \mu$, and 
$S_{3}^{\prime \prime}=\left\{\nu \in \mathfrak{h}^{*} \mid w \cdot \nu<\mu\right.$ for all $\left.w \in W\right\}$. Then all the conclusions (i)-(viii) of Proposition 5.1 hold for these sets of weights, as well.

Proof. The proof is essentially the same as that of Proposition 5.1, but without the difficulties encountered in (vi) and (viii).

Definition 5.9. Let $S_{1}^{\prime}, S_{2}^{\prime}, S_{3}^{\prime}, S_{1}^{\prime \prime}, S_{2}^{\prime \prime}$, and $S_{3}^{\prime \prime}$ be as in Proposition 5.8. We define the translation functors $T_{\lambda_{\alpha}}^{\mu}$ and $T_{\mu}^{\lambda_{\alpha}}$ by $T_{\lambda_{\alpha}}^{\mu} M=$ $W_{0}^{S_{2}^{\prime \prime}}\left(W_{0}^{S_{2}^{\prime}} M \otimes_{K} L(\eta)\right)$ and $T_{\mu}^{\lambda_{\alpha}} M=\left(M^{S_{2}^{\prime \prime}} \otimes_{K} \Gamma(-\eta)\right)^{S_{2}^{\prime}}$. The functor $T_{\lambda_{\alpha}}^{\mu}$ is a functor from the category $C\left(\lambda_{\alpha}\right)$ to the category $C(\mu)$, but the functor $T_{\mu}^{\lambda_{\alpha}}$ is a functor from a subcategory of $C(\mu)$ to the category $C\left(\lambda_{\alpha}\right)$, as it is only defined on those $M$ in $C(\mu)$ with an RHWS which has only finitely many factors of highest weight in $S_{2}^{\prime \prime} \cup S_{3}^{\prime \prime}$.

Once again, the functor $T_{\lambda_{\alpha}}^{\mu}$ can be seen to be right exact. We also have adjoint-like properties, whose proofs are similar to those in Theorems 5.3 and 5.7 and Lemma 5.6, and are left to the reader. The following definition makes their statement easier, and they are summarized in the following theorem.

Definition 5.10. Let $S_{2}^{\prime}$ and $S_{3}^{\prime}$ be as in Proposition 5.8. We define $C\left(\lambda_{\alpha}\right)_{\text {nice }}$ to be the full subcategory of $C\left(\lambda_{\alpha}\right)$ consisting of those modules whose composition factors all have highest weights in $S_{2}^{\prime} \cup S_{3}^{\prime}$.

Theorem 5.11. Let $S_{1}^{\prime}, S_{2}^{\prime}, S_{3}^{\prime}, S_{1}^{\prime \prime}, S_{2}^{\prime \prime}$, and $S_{3}^{\prime \prime}$ be as in Proposition 5.8. If $M \in \mathrm{Ob} C(\mu)$ has a finite $H W S$ with all factors of highest weight in $S_{1}^{\prime \prime} \cup S_{2}^{\prime \prime}$, and if $N \in \mathrm{Ob} C\left(\lambda_{\alpha}\right)_{\text {nice }}$, then there is a natural isomorphism $\operatorname{Hom}\left(T_{\mu}^{\lambda_{\alpha}} M, N\right) \cong \operatorname{Hom}\left(M, T_{\lambda_{\alpha}}^{\mu} N\right)$. If in addition we assume that the finite HWS for $M$ is in fact a finite VS, then there are natural isomorphisms $\operatorname{Ext}_{C\left(\lambda_{\alpha}\right)}^{n}\left(T_{\mu}^{\lambda_{\alpha}} M, N\right) \cong \operatorname{Ext}_{C(\mu)}^{n}\left(M, T_{\lambda_{\alpha}}^{\mu} N\right)$ for each $n=0,1, \ldots$ Also, for any $\nu \in S_{1}^{\prime \prime} \cup S_{2}^{\prime \prime}$, we have that $T_{\mu}^{\lambda_{\alpha}} I^{\mu}(\nu)$ is projective in $C\left(\lambda_{\alpha}\right)_{\text {nice }}$.

We are now able to compose the functors defined thus far in order to obtain the operators of coherent continuation.

DEFINITION 5.12. We define the operators of coherent continuation, $\Theta_{\alpha}=T_{\lambda_{\alpha}}^{\mu} \circ T_{\lambda}^{\lambda_{\alpha}}$ and $\Psi_{\alpha}=T_{\lambda_{\alpha}}^{\lambda} \circ T_{\mu}^{\lambda_{\alpha}}$.

The functor $\Theta_{\alpha}$ is right exact, since it is composed of right exact functors. We may apply the adjoint-like properties proved for the translation functors to obtain similar results for coherent continuation. 
We summarize these results in the following theorem, whose proof is left to the reader.

Theorem 5.13. Let $S_{1}^{\prime}, S_{2}^{\prime}$, and $S_{3}^{\prime}$ be as in Proposition 5.1, and let $S_{1}^{\prime \prime}, S_{2}^{\prime \prime}$, and $S_{3}^{\prime \prime}$ be as in Proposition 5.8. If $M \in \mathrm{Ob} C(\mu)$ has a finite $H W S$ whose factors all have highest weights in $S_{1}^{\prime \prime} \cup S_{2}^{\prime \prime}$, and if $N \in$ $\mathrm{Ob} C(\lambda)_{\text {nice, }}$ then there is a natural isomorphism $\operatorname{Hom}\left(\Psi_{\alpha} M, N\right) \cong$ $\operatorname{Hom}\left(M, \Theta_{\alpha} N\right)$. If we impose the additional hypothesis that $M$ have a finite VS rather than merely an HWS, then we have natural isomorphisms $\operatorname{Ext}_{C(\lambda)}^{n}\left(\Psi_{\alpha} M, N\right) \cong \operatorname{Ext}_{C(\mu)}^{n}\left(M, \Theta_{\alpha} N\right)$ for each $n \geq 0$. Also, for any $\nu \in S_{1}^{\prime \prime} \cup S_{2}^{\prime \prime}$, we have that $\Psi_{\alpha} I^{\mu}(\nu)$ is projective in $C(\lambda)_{\text {nice }}$.

6. The structure of certain translated modules. We retain the notation of the previous section, and study the structure of the translations and coherent continuations of Verma modules and their irreducible quotients. We begin with the behavior of Verma modules under translation and coherent continuation, which involves only a straightforward analysis of the possible resulting Verma factors.

TheOREM 6.1. Let $w \in W$ such that $w<w s$. Then the following hold.

(i) $T_{\lambda}^{\lambda} M(w \cdot \lambda) \cong M\left(w \cdot \lambda_{\alpha}\right) \cong T_{\lambda}^{\lambda} M((w s) \cdot \lambda)$, and furthermore any imbedding of $M((w s) \cdot \lambda)$ into $M(w \cdot \lambda)$ translates to an isomorphism of $T_{\lambda}^{\lambda_{\alpha}} M((w s) \cdot \lambda)$ and $T_{\lambda}^{\lambda_{\alpha}} M(w \cdot \lambda)$.

(ii) There is a short exact sequence

$$
0 \rightarrow M(w \cdot \mu) \rightarrow \Theta_{\alpha} M(w \cdot \lambda) \rightarrow M((w s) \cdot \mu) \rightarrow 0 .
$$

Also, the same is true with $\Theta_{\alpha} M((w s) \cdot \lambda)$ in place of $\Theta_{\alpha} M(w \cdot \lambda)$.

(iii) $T_{\mu}^{\lambda_{\alpha}} M(w \cdot \mu) \cong M\left(w \cdot \lambda_{\alpha}\right) \cong T_{\mu}^{\lambda_{\alpha}} M((w s) \cdot \mu)$, and furthermore any imbedding of $M((w s) \cdot \mu)$ into $M(w \cdot \mu)$ translates to an isomorphism of $T_{\mu}^{\lambda_{\alpha}} M((w s) \cdot \mu)$ and $T_{\mu}^{\lambda_{\alpha}} M(w \cdot \mu)$.

(iv) There is a short exact sequence

$$
0 \rightarrow M(w \cdot \lambda) \rightarrow \Psi_{\alpha} M(w \cdot \mu) \rightarrow M((w s) \cdot \lambda) \rightarrow 0
$$

Also, the same is true with $\Psi_{\alpha} M((w s) \cdot \mu)$ in place of $\Psi_{\alpha} M(w \cdot \mu)$.

(v) The sequences in (ii) and (iv) do not split.

Proof. For the first statement, since $M(w \cdot \lambda) \otimes_{K} L(\eta)$ has a VS, we see that $T_{\lambda}^{\lambda_{\alpha}} M(w \cdot \lambda) \cong\left(M(w \cdot \lambda) \otimes_{K} L(\eta)\right)^{S_{2}^{\prime \prime}}$, where $S_{2}^{\prime \prime}=W \cdot \lambda_{\alpha}$ as in Proposition 5.1. Any factor in the VS for $M(w \cdot \lambda) \otimes_{K} L(\eta)$ has a highest weight of the form $w \cdot \lambda+\nu$, where $\nu \in \Pi(L(\eta))$. In order for 
this highest weight to belong to $S_{2}^{\prime \prime}$, there must be some $x \in W$ with $x \cdot(w \cdot \lambda+\nu)=(x w) \cdot \lambda+x \nu=\lambda_{\alpha}=\lambda+\eta-\alpha$. Since we have $(x w) \cdot \lambda \leq \lambda$ and $x \nu \leq \eta$, we only have two cases to consider. Either $(x w) \cdot \lambda=\lambda$ and $x \nu=\eta-\alpha$, or $(x w) \cdot \lambda=\lambda-\alpha$ and $x \nu=\eta$. In the first case, we have $x w=1$, so that $w=x^{-1}$ and $\nu=x^{-1}(\eta-\alpha)=w(\eta-\alpha)=$ $w s \eta$. In the second case, since $\lambda-\alpha=s \cdot \lambda$, we have $x w=s$, so that $w s=x^{-1}$ and $\nu=x^{-1} \eta=w s \eta$. Thus, the only possible factor in the VS for $T_{\lambda}^{\lambda_{\alpha}} M(w \cdot \lambda) \cong\left(M(w \cdot \lambda) \otimes_{K} L(\eta)\right)^{S_{2}^{\prime \prime}}$ is the Verma module of highest weight $w \cdot \lambda+w s \eta=w \cdot(\lambda+s \eta)=w \cdot(\lambda+\eta-\alpha)=w \cdot \lambda_{\alpha}$. Since $\operatorname{dim} L(\eta)_{w s \eta}=1$, this factor can occur orly once, and we have an isomorphism $T_{\lambda}^{\lambda_{\alpha}} M(w \cdot \lambda) \cong M\left(w \cdot \lambda_{\alpha}\right)$. A similar argument shows that we have an isomorphism $T_{\lambda}^{\lambda_{\alpha}} M((w s) \cdot \lambda) \cong M\left(w \cdot \lambda_{\alpha}\right)$. The proof that any nonzero imbedding $\phi: M((w s) \cdot \lambda) \rightarrow M(w \cdot \lambda)$ translates to an isomorphism $T_{\lambda}^{\lambda_{\alpha}} \phi: T_{\lambda}^{\lambda_{\alpha}} M((w s) \cdot \lambda) \rightarrow T_{\lambda}^{\lambda_{\alpha}} M(w \cdot \lambda)$ is identical to the proof of [7, Lemma 9.5].

To prove the second statement, observe that by (i), we have that $\Theta_{\alpha} M(w \cdot \lambda) \cong T_{\lambda_{\alpha}}^{\mu} M\left(w \cdot \lambda_{\alpha}\right)=\left(M\left(w \cdot \lambda_{\alpha}\right) \otimes_{K} L(\eta)\right)^{S_{2}^{\prime \prime}}$, where $S_{2}^{\prime \prime}=W \cdot \mu$, as in Proposition 5.8, where we use the fact that $M\left(w \cdot \lambda_{\alpha}\right) \otimes_{K} L(\eta)$ has a VS. Now any factor in the VS for $M\left(w \cdot \lambda_{\alpha}\right) \otimes_{K} L(\eta)$ has a highest weight of the form $w \cdot \lambda_{\alpha}+\nu$, where $\nu \in \Pi(L(\eta))$. But then, for any $x \in W$, we have $x \cdot\left(w \cdot \lambda_{\alpha}+\nu\right)=(x w) \cdot \lambda_{\alpha}+x \nu \leq \lambda_{\alpha}+\eta=\mu$, with equality only if both $(x w) \cdot \lambda_{\alpha}=\lambda_{\alpha}$ and $x \nu=\eta$. The first equality only occurs for the two cases $x=w^{-1}$ and $x=s w^{-1}$, so that we have $\nu=w \eta$ or $\nu=w s \eta$. Since $\operatorname{dim} L(\eta)_{w \eta}=\operatorname{dim} L(\eta)_{w s \eta}=1$, we see that $\left(M\left(w \cdot \lambda_{\alpha}\right) \otimes_{K} L(\eta)\right)_{2}^{\prime \prime}$ has exactly two factors in its Verma series, of highest weights $w \cdot \lambda_{\alpha}+w \eta=w \cdot \mu$ and $w \cdot \lambda_{\alpha}+w s \eta=(w s) \cdot \mu$, and each factor occurs once. Since the order of factors in a finite VS may be arranged so that the factors of higher highest weight occur at the bottom of the series, we obtain the existence of the short exact sequence in (ii). Finally, since $\Theta_{\alpha}=T_{\lambda_{\alpha}}^{\mu} \circ T_{\lambda}^{\lambda_{\alpha}}$, we may conclude from (i) that $\Theta_{\alpha} M((w s) \cdot \lambda) \cong \Theta_{\alpha} M(w \cdot \lambda)$.

The proofs of (iii) and (iv) are similar, involving RVS in place of VS, and are left to the reader.

To see that the sequence in (ii) does not split, observe that

$$
\begin{aligned}
\operatorname{dim} & \operatorname{Hom}\left(M((w s) \cdot \mu), \Theta_{\alpha} M(w \cdot \lambda)\right) \\
& =\operatorname{dim} \operatorname{Hom}\left(T_{\mu}^{\lambda_{\alpha}} M((w s) \cdot \mu), T_{\lambda}^{\lambda_{\alpha}} M(w \cdot \lambda)\right) \\
& =\operatorname{dim} \operatorname{Hom}\left(M\left(w \cdot \lambda_{\alpha}\right), M\left(w \cdot \lambda_{\alpha}\right)\right)=1 .
\end{aligned}
$$


Similarly, to see that the sequence in (iv) does not split, observe that

$$
\begin{aligned}
\operatorname{dim} & \operatorname{Hom}\left(\Psi_{\alpha} M(w \cdot \mu), M(w \cdot \lambda)\right) \\
& =\operatorname{dim} \operatorname{Hom}\left(T_{\mu}^{\lambda_{\alpha}} M(w \cdot \mu), T_{\lambda}^{\lambda^{\alpha}} M(w \cdot \lambda)\right) \\
& =\operatorname{dim} \operatorname{Hom}\left(M\left(w \cdot \lambda_{\alpha}\right), M\left(w \cdot \lambda_{\alpha}\right)\right)=1 .
\end{aligned}
$$

We now look at the more complicated behavior of irreducible highest weight modules under translation and coherent continuation.

Proposition 6.2. Let $w \in W$ such that $w<w s$. Then the following hold.

(i) $T_{\lambda}^{\lambda_{\alpha}} L(w \cdot \lambda)=0$.

(ii) $\Theta_{\alpha} L(w \cdot \lambda)=0$.

(iii) $T_{\lambda}^{\lambda_{\alpha}} L((w s) \cdot \lambda)$ is a nonzero highest weight module of weight $w \cdot \lambda_{\alpha}$ in which $L\left(w \cdot \lambda_{\alpha}\right)$ occurs exactly once as a composition factor, and all other composition factors are of the form $L(\nu)$ with $\nu \in S_{3}^{\prime \prime}$, where $S_{3}^{\prime \prime}$ is as in Proposition 5.1.

(iv) There is a unique submodule $L^{\prime}((w s) \cdot \mu)$ of $\Theta_{\alpha} L((w s) \cdot \lambda)$ which is of highest weight $(w s) \cdot \mu$ and in which $L((w s) \cdot \mu)$ occurs exactly once as a composition factor, and all other composition factors are of the form $L(\nu)$ with $\nu \in S_{3}^{\prime \prime}$, where $S_{3}^{\prime \prime}$ is as in Proposition 5.8.

(v) $\operatorname{dim} \operatorname{Hom}\left(\Theta_{\alpha} L((w s) \cdot \lambda), L((w s) \cdot \mu)\right)=1$.

(vi) There is a complex

$$
0 \rightarrow L^{\prime}((w s) \cdot \mu) \stackrel{\phi}{\rightarrow} \Theta_{\alpha} L((w s) \cdot \lambda) \stackrel{\psi}{\rightarrow} L((w s) \cdot \mu) \rightarrow 0
$$

in which $\phi$ is the injective inclusion map, its image being the unique submodule of $\Theta_{\alpha} L((w s) \cdot \lambda)$ with properties given in (iv), and in which $\psi$ is surjective, its image being the unique quotient of $\Theta_{\alpha} L((w s) \cdot \lambda)$ which is irreducible of highest weight $(w s) \cdot \mu$, as given by statement (v).

Proof. For the first statement, consider a short exact sequence

$$
0 \rightarrow M((w s) \cdot \lambda) \stackrel{\phi}{\rightarrow} M(w \cdot \lambda) \rightarrow X \rightarrow 0,
$$

where $\phi$ is any nonzero imbedding of $M((w s) \cdot \lambda)$ into $M(w \cdot \lambda)$, so that $X$ is a highest weight module of weight $w \cdot \lambda$. Since $T_{\lambda}^{\lambda_{\alpha}}$ is defined as a composition of right exact functors, it is right exact, and Proposition 6.1 may be applied to show that $T_{\lambda}^{\lambda_{\alpha}} X=0$. But $L(w \cdot \lambda)$ is a quotient of $X$, so we may apply the right exactness once more to 
obtain $T_{\lambda}^{\lambda}{ }^{\lambda} L(w \cdot \lambda)=0$. The second statement follows immediately from the first.

For the third statement, we have that

$$
T_{\lambda}^{\lambda_{\alpha}} L((w s) \cdot \lambda)=W_{0}^{S_{2}^{\prime \prime}}\left(W_{0}^{S_{2}^{\prime}} L((w s) \cdot \lambda) \otimes_{K} L(\eta)\right),
$$

where $S_{2}^{\prime}$ and $S_{2}^{\prime \prime}$ are as in Proposition 5.1. Using Proposition 2.9 and the definition of the functor $W_{0}^{S_{2}^{\prime \prime}}$, we see that the only possible factors of $T_{\lambda}^{\lambda_{\alpha}} L((w s) \cdot \lambda)$ are of the form $L(\nu)$ with $\nu \in S_{2}^{\prime \prime} \cup S_{3}^{\prime \prime}$. What remains to be shown is that the only possible composition factor of $T_{\lambda}^{\lambda_{\alpha}} L((w s) \cdot \lambda)$ of the form $L\left(x \cdot \lambda_{\alpha}\right)$ is $L\left(w \cdot \lambda_{\alpha}\right)$, and that this factor occurs only once. But, using [6, Theorem 6.9] twice and [6, Corollary 7.5] once, we have

$$
\left(T_{\lambda}^{\lambda_{\alpha}} L((w s) \cdot \lambda): L\left(x \cdot \lambda_{\alpha}\right)\right)=\left(L((w s) \cdot \lambda) \otimes_{K} L(\eta): L\left(x \cdot \lambda_{\alpha}\right)\right) .
$$

Now, [6, Proposition 7.6] states that the only such composition factor which may occur is $L\left(w \cdot \lambda_{\alpha}\right)$, and this factor occurs at most once. On the other hand, using the isomorphism of Theorem 5.3, we have

$$
\begin{aligned}
& \operatorname{dim} \operatorname{Hom}\left(M\left(w \cdot \lambda_{\alpha}\right), T_{\lambda}^{\lambda_{\alpha}} L((w s) \cdot \lambda)\right) \\
& \quad=\operatorname{dim} \operatorname{Hom}\left(T_{\lambda_{\alpha}}^{\lambda} M\left(w \cdot \lambda_{\alpha}\right), L((w s) \cdot \lambda)\right) \\
& \quad=\operatorname{dim} \operatorname{Hom}\left(\Psi_{\alpha} M(w \cdot \mu), L((w s) \cdot \lambda)\right)=1,
\end{aligned}
$$

where we have used the structure of $\Psi_{\alpha} M(w \cdot \mu)$ given by the short exact sequence from Proposition 6.1 for the last equality. Thus $L\left(w \cdot \lambda_{\alpha}\right)$ occurs at least once as a composition factor of $T_{\lambda}^{\lambda} L((w s) \cdot \lambda)$, proving the third statement.

For the fourth statement, we have

$$
\begin{aligned}
& \operatorname{dim} \operatorname{Hom}\left(M((w s) \cdot \mu), \theta_{\alpha} L((w s) \cdot \lambda)\right) \\
& \quad=\operatorname{dim} \operatorname{Hom}\left(\Psi_{\alpha} M((w s) \cdot \mu), L((w s) \cdot \lambda)\right)=1 .
\end{aligned}
$$

We may thus take $L^{\prime}((w s) \cdot \mu)$ to be the unique nonzero image of $M((w s) \cdot \mu)$ in $\Theta_{\alpha} L((w s) \cdot \lambda)$. That is, let

$$
\phi \in \operatorname{Hom}\left(M((w s) \cdot \mu), \Theta_{\alpha} L((w s) \cdot \lambda)\right)
$$

with $\phi \neq 0$, and set $L^{\prime}((w s) \cdot \mu)=\operatorname{Im} \phi$. Clearly $L^{\prime}((w s) \cdot \mu)^{-}$ is a nonzero highest weight module of weight $(w s) \cdot \mu$, and hence $L((w s) \cdot \mu)$ occurs exactly once as a composition factor. It remains to show that $L^{\prime}((w s) \cdot \mu)$ has as its other composition factors only those of highest weight in $S_{3}^{\prime \prime}$. We use the notation $S_{1}^{\prime \prime}, S_{2}^{\prime \prime}$, and 
$S_{3}^{\prime \prime}$ of Proposition 5.8. Let $X$ be the unique maximal submodule of $M((w s) \cdot \mu)$, and consider an HWS for $X$, which exists by [3, Lemma 4.4]. Since, by Proposition 2.9 and Proposition 5.8, the only possible highest weight factors of $X$ have highest weights in $S_{2}^{\prime \prime} \cup S_{3}^{\prime \prime}$, we see by Proposition 3.6 that $X$ has a two step filtration $0 \subset X^{S_{2}^{\prime \prime}} \subset X$, where $X^{S_{2}^{\prime \prime}}$ has an HWS $0=X_{0} \subset X_{1} \subset X_{2} \subset \cdots$ in which each factor has highest weight in $S_{2}^{\prime \prime}$, and where $X / X^{S_{2}^{\prime \prime}}$ has an HWS in which each factor has highest weight in $S_{3}^{\prime \prime}$. We show by induction that each $X_{i} \subset \operatorname{Ker} \phi$. Clearly $X_{0} \subset \operatorname{Ker} \phi$. Assume $X_{i} \subset \phi$. if $X_{i+1} \not \subset \operatorname{Ker} \phi$, then $\phi$ would induce a nonzero map from $X_{i+1} / X_{i}$ to $\Theta_{\alpha} L((w s) \cdot \lambda)$. But then, since by Proposition 2.9 and [4, Theorem 4.1] $X_{i+1} / X_{i}$ has highest weight of the form $x \cdot \mu$ for some $x \in W$ with $x>w s$, we would have a nonzero map from $M(x \cdot \mu)$ to $\Theta_{\alpha} L((w s) \cdot \lambda)$. However, using Proposition 6.1(iii) and (iii) above, we have

$\operatorname{Hom}\left(M(x \cdot \mu), \Theta_{\alpha} L((w s) \cdot \lambda)\right) \cong \operatorname{Hom}\left(T_{\mu}^{\lambda_{\alpha}} M(x \cdot \mu), T_{\lambda}^{\lambda_{\alpha}} L((w s) \cdot \lambda)\right)=0$.

Therefore we must have $X_{i+1} \subset \operatorname{Ker} \phi$, completing the induction, so that in fact $X_{i} \subset \operatorname{Ker} \phi$ for all $i \geq 0$. But $X^{S_{2}^{\prime \prime}}=\bigcup_{i \geq 0} X_{i}$, so we have $X^{S_{2}^{\prime \prime}} \subset \operatorname{Ker} \phi$. This shows that $L^{\prime}((w s) \cdot \mu)=\operatorname{Im} \phi$ is a nonzero quotient of $M((w s) \cdot \mu) / X^{S_{2}^{\prime \prime}}$, and thus $L((w s) \cdot \mu)$ occurs once as a composition factor and all other composition factors are composition factors of $X / X^{\prime \prime} S_{2}^{\prime \prime}$, hence have highest weights in $S_{3}^{\prime \prime}$.

By the right exactness of $\Theta_{\alpha}$, we see that there is an epimorphism $\pi: \Theta_{\alpha} M((w s) \cdot \lambda) \rightarrow \Theta_{\alpha} L((w s) \cdot \lambda)$. Using Proposition 6.1(ii), which gives us a filtration $0 \subset M(w \cdot \lambda) \subset \Theta_{\alpha} M((w s) \cdot \lambda)$, we may consider the images under $\pi$ to obtain a filtration $0 \subset M \subset \Theta_{\alpha} L((w s) \cdot \lambda)$, in which $M$ is either zero or of highest weight $w \cdot \mu$, and in which $\Theta_{\alpha} L((w s) \cdot \lambda) / M$ is either zero or of highest weight $(w s) \cdot \mu$. But

$\operatorname{Hom}\left(M(w \cdot \mu), \Theta_{\alpha} L((w s) \cdot \lambda)\right) \cong \operatorname{Hom}\left(\Psi_{\alpha} M(w \cdot \mu), L((w s) \cdot \lambda)\right) \neq 0$,

so that $M$ is nonzero. To show that $\Theta_{\alpha} L((w s) \cdot \lambda) / M$ is nonzero, note that otherwise we would have $M=\Theta_{\alpha} L((w s) \cdot \lambda)$, and $L(w \cdot \mu)$ would be a quotient of $\Theta_{\alpha} L((w s) \cdot \lambda)$, and hence a quotient of $\Theta_{\alpha} M((w s) \cdot \lambda)$, which would contradict the fact that the sequence in Proposition 6.1(ii) does not split. Therefore $\Theta_{\alpha} L((w s) \cdot \lambda)$ has a nonzero quotient $\Theta_{\alpha} L((w s) \cdot \lambda) / M$ of highest weight $(w s) \cdot \mu$, which in turn has $L((w s) \cdot \mu)$ as a quotient, which shows that

$$
\operatorname{Hom}\left(\Theta_{\alpha} L((w s) \cdot \lambda), L((w s) \cdot \mu)\right) \neq 0 \text {. }
$$

On the other hand, since $\Theta_{\alpha} L((w s) \cdot \lambda)$ is a quotient of $\Theta_{\alpha} M((w s) \cdot \lambda)$, we have 


$$
\begin{aligned}
& \operatorname{dim} \operatorname{Hom}\left(\Theta_{\alpha} L((w s) \cdot \lambda), L((w s) \cdot \mu)\right) \\
& \quad \leq \operatorname{dim} \operatorname{Hom}\left(\Theta_{\alpha} M((w s) \cdot \lambda), L((w s) \cdot \mu)\right)=1,
\end{aligned}
$$

by Proposition 6.1(ii). This proves (v).

Finally, the only part of (vi) which does not follow immediately from (iv) and (v) is that $\operatorname{Im} \phi \subset \operatorname{Ker} \psi$. Let $i: M((w s) \cdot \mu) \rightarrow M(w \cdot \mu)$ be a nonzero imbedding. By Proposition 6.1(iii), this translates to an isomorphism $T_{\mu}^{\lambda_{\alpha}} i: M\left(w \cdot \lambda_{\alpha}\right) \rightarrow M\left(w \cdot \lambda_{\alpha}\right)$. We now have a square

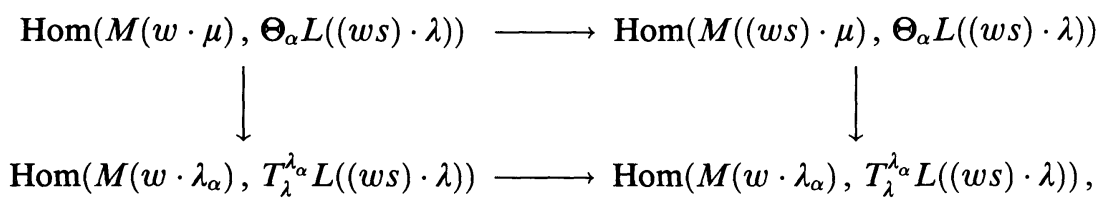

where the vertical maps are the natural isomorphisms of Theorem 5.11 , the top horizontal map is just the restriction map given by transposition of $i$, and the bottom horizontal map is given by the transposition of the isomorphism $T_{\mu}^{\lambda^{\alpha}} i$, so that the bottom horizontal map is also an isomorphism. Note that the naturality implies that this square commutes. By (iii), the vector spaces on the bottom of this diagram are 1-dimensional, hence so are the ones on top. The commutativity shows that the top map must be an isomorphism, and hence any nonzero map from $M(w \cdot \mu)$ to $\Theta_{\alpha} L((w s) \cdot \lambda)$ has a nonzero restriction to $M((w s) \cdot \mu)$, and hence its image $N$, which is a highest weight module of weight $w \cdot \mu$, contains a nonzero image of $M((w s) \cdot \mu)$, which is $L^{\prime}((w s) \cdot \mu)$ by (iv). Now consider the filtration $0 \subset M \subset \Theta_{\alpha} L((w s) \cdot \lambda)$ from the proof of $(\mathrm{v})$. It is clear that $M$ is the unique submodule of highest weight $w \cdot \mu$, so that $M=N$, and hence $L^{\prime}((w s) \cdot \mu) \subset M$. On the other hand, from the proof of (v) we have $M \subset \operatorname{Ker} \psi$, so that $\operatorname{Im} \phi=L^{\prime}((w s) \cdot \mu) \subset M \subset \operatorname{Ker} \psi$.

We may now define certain submodules which have been studied extensively in the case of a finite dimensional algebra.

Definition 6.3. We use the notation of Proposition 6.2(vi). We define $U_{\alpha} L((w s) \cdot \lambda)=\operatorname{Ker} \psi / \operatorname{Im} \phi, K_{\alpha} L((w s) \cdot \lambda)=\operatorname{Ker} \psi$, and $Q_{\alpha} L((w s) \cdot \lambda)=\Theta_{\alpha} L((w s) \cdot \lambda) / \operatorname{Im} \phi$.

Observe that we have short exact sequences

$$
\begin{aligned}
& 0 \rightarrow L^{\prime}((w s) \cdot \mu) \rightarrow K_{\alpha} L((w s) \cdot \lambda) \rightarrow U_{\alpha} L((w s) \cdot \lambda) \rightarrow 0 \\
& 0 \rightarrow L^{\prime}((w s) \cdot \mu) \rightarrow \theta_{\alpha} L((w s) \cdot \lambda) \rightarrow Q_{\alpha} L((w s) \cdot \lambda) \rightarrow 0 \\
& 0 \rightarrow U_{\alpha} L((w s) \cdot \lambda) \rightarrow Q_{\alpha} L((w s) \cdot \lambda) \rightarrow L((w s) \cdot \mu) \rightarrow 0
\end{aligned}
$$


and

$$
0 \rightarrow K_{\alpha} L((w s) \cdot \lambda) \rightarrow \Theta_{\alpha} L((w s) \cdot \lambda) \rightarrow L((w s) \cdot \mu) \rightarrow 0 .
$$

We now take a look at what composition factors of the form $L(x \cdot \mu)$ can occur in $U_{\alpha} L((w s) \cdot \lambda)$. For this, we first need a lemma involving a further translation. Note that $\mu$ satisfies exactly the same hypotheses as $\lambda$, namely that $\mu \in P^{+}$and $\mu\left(h_{i}\right)=0$. Thus we may set $\mu_{\alpha}=$ $\mu+\eta$ and define the translation functors $T_{\mu}^{\mu_{\alpha}}$ and $T_{\mu_{\alpha}}^{\mu}$ exactly as in Definition 5.2. These translation functors have all the same properties as $T_{\lambda}^{\lambda_{\alpha}}$ and $T_{\lambda_{\alpha}}^{\lambda}$.

LEMMA 6.4. For any $x \in W$ and any short exact sequence

$$
0 \rightarrow L \rightarrow M \rightarrow N \rightarrow 0
$$

of modules in $C(\mu)_{\text {nice, }}\left(T_{\mu}^{\mu_{\alpha}} M: L\left(x \cdot \mu_{\alpha}\right)\right)=\left(T_{\mu}^{\mu_{\alpha}} L: L\left(x \cdot \mu_{\alpha}\right)\right)+$

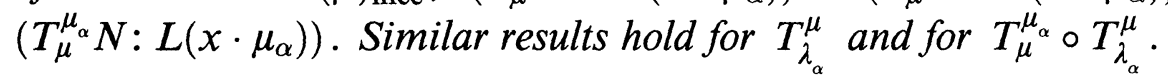

Proof. Since $T_{\mu_{\alpha}}^{\mu} I^{\mu_{\alpha}}\left(x \cdot \mu_{\alpha}\right)$ is projective in $C(\mu)_{\text {nice }}$ by Lemma 5.6, we have that the sequence

$$
\begin{aligned}
0 & \rightarrow \operatorname{Hom}\left(T_{\mu_{\alpha}}^{\mu} I^{\mu_{\alpha}}\left(x \cdot \mu_{\alpha}\right), L\right) \rightarrow \operatorname{Hom}\left(T_{\mu_{\alpha}}^{\mu} I^{\mu_{\alpha}}\left(x \cdot \mu_{\alpha}\right), M\right) \\
& \rightarrow \operatorname{Hom}\left(T_{\mu_{\alpha}}^{\mu} I^{\mu_{\alpha}}\left(x \cdot \mu_{\alpha}\right), N\right) \rightarrow 0
\end{aligned}
$$

is exact. We now use Theorem 5.3 and Proposition 2.7 repeatedly to obtain

$$
\begin{aligned}
\left(T_{\mu}^{\mu_{\alpha}} M: L\left(x \cdot \mu_{\alpha}\right)\right)=\operatorname{dim} \operatorname{Hom}\left(I^{\mu_{\alpha}}\left(x \cdot \mu_{\alpha}\right), T_{\mu}^{\mu_{\alpha}} M\right) \\
\quad=\operatorname{dim} \operatorname{Hom}\left(T_{\mu_{\alpha}}^{\mu} I^{\mu_{\alpha}}\left(x \cdot \mu_{\alpha}\right), M\right) \\
\quad=\operatorname{dim} \operatorname{Hom}\left(T_{\mu_{\alpha}}^{\mu} I^{\mu_{\alpha}}\left(x \cdot \mu_{\alpha}\right), L\right)+\operatorname{dim} \operatorname{Hom}\left(T_{\mu_{\alpha}}^{\mu} I_{\alpha}^{\mu_{\alpha}}\left(x \cdot \mu_{\alpha}\right), N\right) \\
\quad=\operatorname{dim} \operatorname{Hom}\left(I_{\alpha}^{\mu_{\alpha}}\left(x \cdot \mu_{\alpha}\right), T_{\mu}^{\mu_{\alpha}} L\right)+\operatorname{dim} \operatorname{Hom}\left(I^{\mu_{\alpha}}\left(x \cdot \mu_{\alpha}\right), T_{\mu}^{\mu_{\alpha}} N\right) \\
\quad=\left(T_{\mu}^{\mu_{\alpha}} L: L\left(x \cdot \mu_{\alpha}\right)\right)+\left(T_{\mu}^{\mu_{\alpha}} N: L\left(x \cdot \mu_{\alpha}\right)\right) .
\end{aligned}
$$

The above lemma essentially says that although the translation functor $T_{\mu}^{\mu_{\alpha}}$ may not be exact, its lack of exactness involves only modules with composition factors whose highest weights are in "lower" orbits, and that they behave like exact functors if we consider composition factors of highest weight in $W \cdot \mu_{\alpha}$. Similar statements hold for the other translation functors and compositions of these functors.

Corollary 6.5. For any $x \in W$ and any $M \in \mathrm{Ob} C\left(\lambda_{\alpha}\right)_{\text {nice }},\left(T_{\mu}^{\mu_{\alpha} \circ}\right.$ $\left.T_{\lambda_{\alpha}}^{\mu} M: L\left(x \cdot \mu_{\alpha}\right)\right)=2\left(M: L\left(x \cdot \lambda_{\alpha}\right)\right)$. 
Proof. First note that by the exactness of translation functors on modules with VS as in [6, Theorem 4.8], we may use Proposition

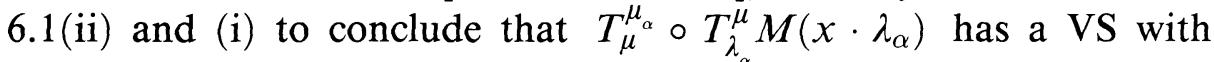
just two factors, each isomorphic to $M\left(x \cdot \mu_{\alpha}\right)$. This implies that

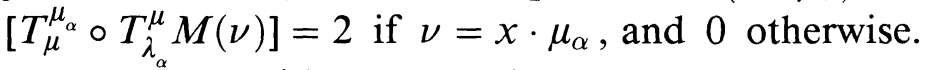

We now consider a resolution

$$
\cdots \rightarrow M_{1} \rightarrow M_{0} \rightarrow M \rightarrow 0
$$

of $M$ by modules in $C\left(\lambda_{\alpha}\right)_{\text {nice }}$ which each have a VS, as constructed in Lemma 4.3. Note that if we use the standard resolution of $K$ when performing this construction, we can guarantee that $M_{n}$ has

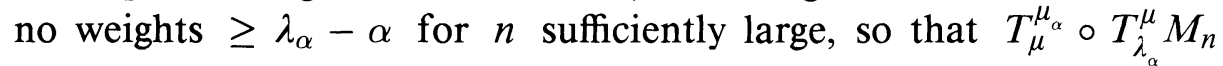
has no weights $\geq \mu_{\alpha}$. Thus the following sums, which may appear to be infinite, have only finitely many nonzero terms. Also, since $y \cdot \mu_{\alpha}=(y s) \cdot \mu_{\alpha}$ and $y \cdot \lambda_{\alpha}=(y s) \cdot \lambda_{\alpha}$ for any $y \in W$, we avoid repeated terms in the following sums by assuming $y<y s$. Applying Lemma 6.4 gives

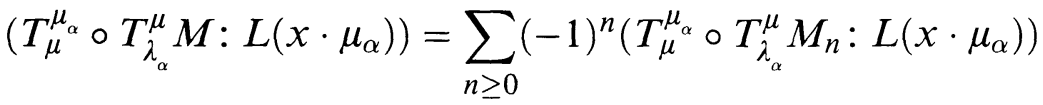

$$
\begin{aligned}
& =\sum_{n \geq 0}(-1)^{n} \sum_{\substack{y \in W \\
y<y s}}\left[T_{\mu}^{\mu_{\alpha}} \circ T_{\lambda_{\alpha}}^{\mu} M_{n}: M\left(y \cdot \mu_{\alpha}\right)\right]\left(M\left(y \cdot \mu_{\alpha}\right): L\left(x \cdot \mu_{\alpha}\right)\right) \\
& =\sum_{n \geq 0}(-1)^{n} \sum_{\substack{y \in W \\
y<y s}} \sum_{\substack{z \in W \\
z<z s}}\left[M_{n}: M\left(z \cdot \lambda_{\alpha}\right)\right]
\end{aligned}
$$

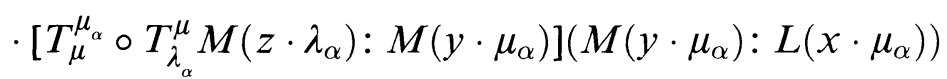

$$
\begin{aligned}
& =\sum_{n \geq 0}(-1)^{n} \sum_{\substack{y \in W \\
y<y s}} 2\left[M_{n}: M\left(y \cdot \lambda_{\alpha}\right)\right]\left(M\left(y \cdot \mu_{\alpha}\right): L\left(x \cdot \mu_{\alpha}\right)\right) \\
& =\sum_{n \geq 0}(-1)^{n} \sum_{\substack{y \in W \\
y<y s}} 2\left[M_{n}: M\left(y \cdot \lambda_{\alpha}\right)\right]\left(M\left(y \cdot \lambda_{\alpha}\right): L\left(x \cdot \lambda_{\alpha}\right)\right) \\
& =2\left(M: L\left(x \cdot \lambda_{\alpha}\right)\right),
\end{aligned}
$$

where we have used [6, Theorem 7.10] for the penultimate equality. Note that we have relied heavily on Propositions 2.9, 5.1 and 5.8 to justify only considering highest weights in the appropriate orbits and: ignoring highest weights in one of the sets of the type $S_{3}$.

THEOREM 6.6. The only composition factors of $U_{\alpha} L((w s) \cdot \lambda)$ which are of the form $L(x \cdot \mu)$ with $x \in W$ satisfy $x<x s$, with $L(w \cdot \mu)$ 
occurring exactly once. Using the sets $S_{1}^{\prime \prime}, S_{2}^{\prime \prime}$, and $S_{3}^{\prime \prime}$ as in Proposition 5.8, all other composition factors of $U_{\alpha} L((w s) \cdot \lambda)$ have highest weights in $S_{3}^{\prime \prime}$.

Proof. We first look at the composition factors of $\Theta_{\alpha} L((w s) \cdot \lambda)$. From the filtration $0 \subset M \subset \Theta_{\alpha} L((w s) \cdot \lambda)$ in the proof of Proposition $6.2(\mathrm{v})$, we see that $L(w \cdot \mu)$ occurs as a composition factor exactly once, and all composition factors have highest weights either in $S_{2}^{\prime \prime}=W \cdot \mu$ or in $S_{3}^{\prime \prime}$. Also, from Proposition 6.2(vi), we see that the composition factor $L((w s) \cdot \mu)$ occurs at least twice. Now we have by Proposition 6.2(iii) and Corollary 6.5 that the only composition factor of $T_{\mu}^{\mu_{\alpha}} \Theta_{\alpha} L((w s) \cdot \lambda)$ of the form $L\left(x \cdot \mu_{\alpha}\right)$ is $L\left(w \cdot \mu_{\alpha}\right)$, which occurs exactly twice. But, applying $T_{\mu}^{\mu_{\alpha}}$ to the individual composition factors of $\Theta_{\alpha} L((w s) \cdot \lambda)$ and using Lemma 6.4, we see that the two factors $L((w s) \cdot \mu)$ translate to the factors each having a composition factor $L\left(w \cdot \mu_{\alpha}\right)$ by Proposition 6.2(iii), so that all other composition factors of $\boldsymbol{\Theta}_{\alpha} L((w s) \cdot \lambda)$ of the form $L(x \cdot \mu)$ must vanish under the translation $T_{u}^{\mu_{\alpha}}$. Thus, by Proposition 6.2(i) and (iii) we see that these composition factors must satisfy $x<x s$. We now use Proposition 6.2(vi) to eliminate the composition factors of $L^{\prime}((w s) \cdot \mu)$ and $L((w s) \cdot \mu)$, obtaining the desired result for $U_{\alpha} L((w s) \cdot \lambda)$.

7. The main theorem. We continue with the notation of the preceding section. We now use the modules $U_{\alpha} L((w s) \cdot \lambda)$ defined in that section to show that, assuming certain nice properties which hold in the case of a finite dimensional algebra carry over to our present situation, we may compute the integers $\operatorname{dim} \operatorname{Ext}_{C(\mu)}^{n}(M(x \cdot \mu), L(y \cdot \mu))$ for any $x, y \in W$, using induction on $l(x)$. Note that these integers were shown to be independent of the choice of $\mu \in P^{+}$in [7, Theorem 8.6]. Also, knowing these integers allows us to compute the multiplicities $[L(y \cdot \mu): M(x \cdot \mu)]$, by [7, Proposition 10.3], and then, using Möbius inversion, the multiplicities $(M(y \cdot \mu): L(x \cdot \mu))$.

LEMMA 7.1. If $x<x s$ and $y<y s$, then $\operatorname{Ext}_{C(\lambda)}^{n}(M(x \cdot \lambda), L(y \cdot \lambda))$ $\cong \operatorname{Ext}_{C(\lambda)}^{n+1}(M((x s) \cdot \lambda), L(y \cdot \lambda))$ for all $n \geq 0$.

Proof. Using the short exact sequence

$$
0 \rightarrow M(x \cdot \lambda) \rightarrow \Psi_{\alpha} M(x \cdot \mu) \rightarrow M((x s) \cdot \lambda) \rightarrow 0
$$

from Proposition 6.1(iv), we apply the functor $\operatorname{Hom}(-, L(y \cdot \lambda))$ to obtain a long exact sequence, and we see that it suffices to show that 
$\operatorname{Ext}_{C(\lambda)}^{n}\left(\Psi_{\alpha} M(x \cdot \mu), L(y \cdot \lambda)\right)=0$ for all $n \geq 0$. But this is clear, since $\operatorname{Ext}_{C(\lambda)}^{n}\left(\Psi_{\alpha} M(x \cdot \mu), L(y \cdot \lambda)\right) \cong \operatorname{Ext}_{C(\lambda)}^{n}\left(M(x \cdot \mu), \Theta_{\alpha} L(y \cdot \lambda)\right)$ by Theorem 5.13 and $\Theta_{\alpha} L(y \cdot \lambda)=0$ by Proposition 6.2(ii).

The following two lemmas will form the heart of our argument, and are essentially an adaptation of the proof of [9, Theorem 3.5].

LEMMA 7.2. If $x<x s$ and $y<y s$, then

$$
\operatorname{Ext}_{C(\mu)}^{n}\left(M(x \cdot \mu), K_{\alpha} L((y s) \cdot \lambda)\right) \cong \operatorname{Ext}_{C(\lambda)}^{n}(M((x s) \cdot \lambda), L((y s) \cdot \lambda))
$$

for all $n \geq 0$.

Proof. Consider the diagram

$$
\begin{aligned}
\ldots \rightarrow \operatorname{Ext}_{C(\mu)}^{n}\left(M(x \cdot \mu), K_{\alpha} L((y s) \cdot \lambda)\right) & \rightarrow \operatorname{Ext}_{C(\mu)}^{n}\left(M(x \cdot \mu), \Theta_{\alpha} L((y s) \cdot \lambda)\right) \\
& \downarrow \cong \operatorname{Ext}_{C(\lambda)}^{n}(M((x s) \cdot \lambda), L((y s) \cdot \lambda)) \\
& \rightarrow \operatorname{Ext}_{C(\lambda)}^{n}\left(\Psi_{\alpha} M(x \cdot \mu), L((y s) \cdot \lambda)\right) \\
& \rightarrow \operatorname{Ext}_{C(\mu)}^{n}(M(x \cdot \mu), L((y s) \cdot \mu)) \rightarrow \cdots \\
& \downarrow \cong \operatorname{Ext}_{C(\lambda)}^{n}(M(x \cdot \lambda), L((y s) \cdot \lambda)) \rightarrow \cdots
\end{aligned}
$$

where the top row is a long exact sequence derived from the short exact sequence

$$
0 \rightarrow K_{\alpha} L((y s) \cdot \lambda) \rightarrow \Theta_{\alpha} L((y s) \cdot \lambda) \rightarrow L((y s) \cdot \mu) \rightarrow 0
$$

which was noted after Definition 6.3, and the bottom row is a long exact sequence derived from the short exact sequence

$$
0 \rightarrow M(x \cdot \lambda) \rightarrow \Psi_{\alpha} M(x \cdot \mu) \rightarrow M((x s) \cdot \lambda) \rightarrow 0
$$

of Proposition 6.1(iv). The vertical maps are the natural isomorphisms of Theorem 5.13 and [7, Proposition 8.5]. Notice that the first vertical isomorphism is essentially derived from the natural equivalence of functors

$$
\operatorname{Hom}(-,-\otimes L(\eta) \otimes L(\eta)) \quad \text { and } \operatorname{Hom}(-\otimes \Gamma(-\eta) \otimes \Gamma(-\eta),-),
$$

and the second from the natural equivalence of functors

$$
\operatorname{Hom}(-,-\otimes L(\mu-\lambda)) \text { and } \operatorname{Hom}(-\otimes \Gamma(\lambda-\mu),-) \text {, }
$$

as proved in [7, Lemma 7.7]. Since $L(\mu-\lambda)$ is a subquotient of $L(\eta) \otimes$ $L(\eta)$, the naturality shows that the diagram commutes up to a scalar multiple, where the scalar depends on our choices for highest weight 
vectors in the subquotient modules. By the commutativity up to scalar multiple, we may define, noncanonically, the desired isomorphisms.

LEMMA 7.3. For any $w<w s$ and $z<z s$, we have a long exact sequence

$$
\begin{aligned}
\cdots & \rightarrow \operatorname{Ext}_{C(\mu)}^{n}(M(z \cdot \mu), L((w s) \cdot \mu)) \rightarrow \operatorname{Ext}_{C(\mu)}^{n}(M((z s) \cdot \mu), L((w s) \cdot \mu)) \\
& \rightarrow \operatorname{Ext}_{C(\mu)}^{n}\left(M(z \cdot \mu), U_{\alpha} L((w s) \cdot \lambda)\right) \rightarrow \cdots .
\end{aligned}
$$

Proof. Using the short exact sequence

$$
0 \rightarrow L^{\prime}((w s) \cdot \mu) \rightarrow K_{\alpha} L((w s) \cdot \lambda) \rightarrow U_{\alpha} L((w s) \cdot \lambda) \rightarrow 0
$$

which was noted after Definition 6.3, we obtain a long exact sequence

$$
\begin{aligned}
\cdots & \rightarrow \operatorname{Ext}_{C(\mu)}^{n}\left(M(z \cdot \mu), L^{\prime}((w s) \cdot \mu)\right) \rightarrow \operatorname{Ext}_{C(\mu)}^{n}\left(M(z \cdot \mu), K_{\alpha} L((w s) \cdot \lambda)\right) \\
& \rightarrow \operatorname{Ext}_{C(\mu)}^{n}\left(M(z \cdot \mu), U_{\alpha} L((w s) \cdot \lambda)\right) \rightarrow \cdots .
\end{aligned}
$$

From the description of the composition factors of $L^{\prime}((w s) \cdot \mu)$ given in Proposition 6.2(iv), we see by Propositions 2.9 and 5.8 that

$$
\operatorname{Ext}_{C(\mu)}^{n}\left(M(z \cdot \mu), L^{\prime}((w s) \cdot \mu)\right) \cong \operatorname{Ext}_{C(\mu)}^{n}(M(z \cdot \mu), L((w s) \cdot \mu)),
$$

and substituting this in the long exact sequence gives

$$
\begin{aligned}
\cdots & \rightarrow \operatorname{Ext}_{C(\mu)}^{n}(M(z \cdot \mu), L((w s) \cdot \mu)) \rightarrow \operatorname{Ext}_{C(\mu)}^{n}\left(M(z \cdot \mu), K_{\alpha} L((w s) \cdot \lambda)\right) \\
& \rightarrow \operatorname{Ext}_{C(\mu)}^{n}\left(M(z \cdot \mu), U_{\alpha} L((w s) \cdot \lambda)\right) \rightarrow \cdots .
\end{aligned}
$$

Making a further substitution from Lemma 7.2 gives

$$
\begin{aligned}
\cdots & \rightarrow \operatorname{Ext}_{C(\mu)}^{n}(M(z \cdot \mu), L((w s) \cdot \mu)) \rightarrow \operatorname{Ext}_{C(\lambda)}^{n}(M((z s) \cdot \lambda), L((w s) \cdot \lambda)) \\
& \rightarrow \operatorname{Ext}_{C(\mu)}^{n}\left(M(z \cdot \mu), U_{\alpha} L((w s) \cdot \lambda)\right) \rightarrow \cdots .
\end{aligned}
$$

Finally, applying [7, Theorem 8.6] on the independence of the choice of dominant integral weight gives the desired result.

In order to use the long exact sequence of Lemma 7.3 in our inductive scheme, we will need the following two properties, which hold in the case of a finite dimensional algebra.

Conjecture 7.4. Let $x, y \in W$. Then the following hold.

(i) If $\operatorname{Ext}_{C(\mu)}^{n}(M(x \cdot \mu)) \neq 0$, then $l(x)-l(y) \equiv n(\bmod 2)$.

(ii) If $x<x s$ and $y<y s$ and $L(x \cdot \mu)$ occurs as a composition factor of $U_{\alpha} L((y s) \cdot \lambda)$, then $\operatorname{Hom}\left(M(x \cdot \mu), U_{\alpha} L((y s) \cdot \lambda)\right) \neq 0$. 
REMARK. The second part of the above conjecture is actually a weaker form of [9, Conjecture 2.5], where it was conjectured, and later proved in [10, Corollary 7.18], that $U_{\alpha} L((y s) \cdot \lambda)$ is completely reducible.

Lemma 7.5. Let $z<z s$ and $w<w s$. Assuming Conjecture 7.4, if $L(z \cdot \mu)$ occurs as a composition factor of $U_{\alpha} L((w s) \cdot \lambda)$, then $l(z) \equiv$ $l(w)(\bmod 2)$.

Proof. Using Conjecture 7.4(ii), we have

$$
\operatorname{Hom}\left(M(z \cdot \mu), U_{\alpha} L((w s) \cdot \mu)\right) \neq 0,
$$

so that by the long exact sequence in Lemma 7.3 we have either $\operatorname{Hom}(M((z s) \cdot \mu), L((w s) \cdot \mu)) \neq 0$ or $\operatorname{Ext}_{C(\mu)}^{1}(M(z \cdot \mu), L((w s) \cdot \mu)) \neq$ 0 , and in either case the result follows from Conjecture 7.4(i).

Lemma 7.6. Assume that Conjecture 7.4 holds. Let $x<x s$ and $y<y s$. If $l(x)-l(y) \not \equiv n(\bmod 2)$, then

$$
\operatorname{Ext}_{C(\mu)}^{n}\left(M(x \cdot \mu), U_{\alpha} L((y s) \cdot \lambda)\right)=0 .
$$

On the other hand, if $l(x)-l(y) \equiv n(\bmod 2)$, then

$$
\begin{aligned}
\operatorname{dim} & \operatorname{Ext}_{C(\mu)}^{n}\left(M(x \cdot \mu), U_{\alpha} L((y s) \cdot \lambda)\right) \\
& =\sum_{z \in W}\left(U_{\alpha} L((y s) \cdot \lambda): L(z \cdot \mu)\right) \operatorname{dim} \operatorname{Ext}_{C(\mu)}^{n}(M(x \cdot \mu), L(z \cdot \mu)) .
\end{aligned}
$$

Proof. Consider a local composition series for $U_{\alpha} L((y s) \cdot \lambda)$ at $x \cdot \mu$. In case $l(x)-l(y) \not \equiv n(\bmod 2)$, we have that

$$
\operatorname{Ext}_{C(\mu)}^{n}(M(x \cdot \mu), F)=0
$$

for each factor $F$ in the LCS, by Lemma 7.5, and hence

$$
\operatorname{Ext}_{C(\mu)}^{n}\left(M(x \cdot \mu), U_{\alpha} L((y s) \cdot \lambda)\right)=0,
$$

by induction on the number of factors. The case where $l(x)-l(y) \equiv n$ (mod 2) also follows by induction on the number of factors, since Conjecture 7.4(i) shows that both $\operatorname{Ext}_{C(\mu)}^{n-1}(M(x \cdot \mu), F)$ and $\operatorname{Ext}_{C(\mu)}^{n+1}(M(x \cdot \mu), F)$ for each factor $F$ in the LCS.

Corollary 7.7. Assume that Conjecture 7.4 holds, and let $x<x s$ and $y<y s$. Then

$$
\operatorname{dim} \operatorname{Hom}\left(M(x \cdot \mu), U_{\alpha} L((y s) \cdot \lambda)\right)=\left(U_{\alpha} L((y s) \cdot \lambda): L(x \cdot \mu)\right) .
$$


Proof. This follows immediately from Lemma 7.6, with $n=0$, when we observe that $\operatorname{Hom}(M(x \cdot \mu), L(z \cdot \mu))=0$ unless $x=z$.

Definition 7.8. We define the polynomials $\bar{P}_{x, y}(q) \in \mathbb{Z}\left[q^{1 / 2}\right]$ for each $x, y \in W$ by

$$
\bar{P}_{x, y}(q)=\sum_{n \geq 0} \operatorname{dim} \operatorname{Ext}_{C(\mu)}^{n}(M(x \cdot \mu), L(y \cdot \mu)) q^{(l(x)-l(y)-n) / 2} .
$$

REMARKS. If we assume the truth of Conjecture 7.4, then we have that $\bar{P}_{x, y}(q) \in \mathbf{Z}[q]$, and it was shown in [7, Corollary 10.4] that we have $[L(y \cdot \lambda): M(x \cdot \lambda)]=(-1)^{l(x)-l(y)} \bar{P}_{x, y}(1)$. Thus, determining the Ext dimensions determines the multiplicities $[L(y \cdot \lambda): M(x \cdot \lambda)]$ and hence, by Möbius inversion, the multiplicities $(M(y \cdot \lambda): L(x \cdot \lambda))$.

For the case of a finite dimensioanl algebra, it was shown in [10, Theorem 7.3] that $\bar{P}_{x, y}$ is the same as the Kazhdan-Lusztig polynomial $P_{x w_{0}, y w_{0}}$, where $w_{0}$ is the longest element in $W$. In the present situation, we have no longest element of $W$ to work with, and we begin our inductive scheme with the dominant integral weight $\mu$ by starting with $x=1$, rather than beginning with the weight $w_{0} \cdot \mu$ as in the proof for the finite dimensional case.

Definition 7.9. For any $x \neq y$, we define

$$
\mu(x, y)=\operatorname{dim} \operatorname{Ext}_{C(\mu)}^{1}(M(x \cdot \mu), L(y \cdot \mu)) .
$$

We also define $\mu(x, x)=1$ for any $x \in W$.

Remark. Assume $x \neq y$. Since $\operatorname{Hom}(M(x \cdot \mu), L(y \cdot \mu))=0$, we have that $\operatorname{deg} \bar{P}_{x, y} \leq(l(x)-l(y)-1) / 2$, so that $\mu(x, y)$ is the leading coefficient of $\bar{P}_{x, y}$ when it has maximal possible degree $(l(x)-l(y)-1) / 2$, and is zero otherwise.

Corollary 7.10. Assume that Conjecture 7.4 holds, and let $z<z s$ and $y<y s$ with $z \neq y$. Then $\left(U_{\alpha} L((y s) \cdot \lambda): L(z \cdot \mu)\right)=\mu(z, y s)$.

Proof. Since $z \neq y$, we also have $z s \neq y s$, and hence

$$
\operatorname{Hom}(M((z s) \cdot \mu), L((y s) \cdot \mu))=0 \text {. }
$$

Also, for either side to be nonzero, we can assume that $l(z) \equiv l(y)$ (mod 2), by Lemma 7.5 and Conjecture 7.4(i). But then, using Conjecture 7.4(i) again, $\operatorname{Ext}_{C(\mu)}^{1}(M((z s) \cdot \mu), L((y s) \cdot \mu))=0$, so by the long exact sequence in Lemma 7.3 we have

$$
\begin{aligned}
& \operatorname{dim} \operatorname{Hom}\left(M(z \cdot \mu), U_{\alpha} L((y s) \cdot \lambda)\right) \\
& \quad=\operatorname{dim} \operatorname{Ext}_{C(\mu)}^{1}(M(z \cdot \mu), L((y s) \cdot \mu))=\mu(z, y s) .
\end{aligned}
$$


TheOREM 7.11. Let $\mu \in P^{+}$, and assume the truth of Conjecture 7.4. The integers $\operatorname{dim} \operatorname{Ext}_{C(\mu)}^{n}(M(x \cdot \mu), L(y \cdot \mu))$ can be computed by induction on $l(x)$ as follows. When $x=1$, we have

$$
\operatorname{dim} \operatorname{Ext}_{C(\mu)}^{n}(M(\mu), L(y \cdot \mu))=0
$$

unless $y=1$ and $n=0$, and $\operatorname{dim} \operatorname{Hom}(M(\mu), L(\mu))=1$. For $x \neq 1$, choose a simple reflection $s$ such that $x s<x$. We then have the following two cases.

Case 1. $y<y$ s. Then $\operatorname{dim} \operatorname{Ext}_{C(\mu)}^{n}(M(x \cdot \mu), L(y \cdot \mu))=\operatorname{dim} \operatorname{Ext}_{C(\mu)}^{n-1}(M((x s) \cdot \mu), L(y \cdot \mu))$.

Case 2. $y>y s$. Then when $l(x)-l(y) \not \equiv n(\bmod 2)$ we have $\operatorname{dim} \operatorname{Ext}_{C(\mu)}^{n}(M(x \cdot \mu), L(y \cdot \mu))=0$, and when $l(x)-l(y) \equiv n(\bmod 2)$ we have

$$
\begin{aligned}
\operatorname{dim} \operatorname{Ext}_{C(\mu)}^{n}(M(x \cdot \mu), L(y \cdot \mu)) \\
=\operatorname{dim} \operatorname{Ext}_{C(\mu)}^{n}(M((x s) \cdot \mu), L((y s) \cdot \mu)) \\
\quad+\sum_{\substack{z \in W \\
z<z s}} \mu(z, y) \operatorname{dim} \operatorname{Ext}_{C(\mu)}^{n}(M((x s) \cdot \mu), L(z \cdot \mu)) \\
\quad-\operatorname{dim}_{\operatorname{Ext}_{C(\mu)}^{n+1}(M((x s) \cdot \mu), L(y \cdot \mu))}
\end{aligned}
$$

Proof. The assertions for $x=1$ are clear from Proposition 2.9, so we choose a simple reflection $s$ with $x s<x$, and choose $\lambda$ and $\mu$ as in the preceding two sections. Case 1 is simply a restatement of Lemma 7.1. For Case 2, we can assume by the parity conjecture, i.e. Conjecture 7.4 that $l(x)-l(y) \equiv n(\bmod 2)$, and we use the long exact sequence

$$
\begin{aligned}
\cdots & \rightarrow \operatorname{Ext}_{C(\mu)}^{n}(M((x s) \cdot \mu), L(y \cdot \mu)) \rightarrow \operatorname{Ext}_{C(\mu)}^{n}(M(x \cdot \mu), L(y \cdot \mu)) \\
& \rightarrow \operatorname{Ext}_{C(\mu)}^{n}\left(M((x s) \cdot \mu), U_{\alpha} L(y \cdot \lambda)\right) \rightarrow \cdots
\end{aligned}
$$

of Lemma 7.3. By the parity conjecture and Lemma 7.6, this long exact sequence collapses into a family of short exact sequences, and we have

$$
\begin{aligned}
\operatorname{dim} \operatorname{Ext}_{C(\mu)}^{n}(M(x \cdot \mu), L(y \cdot \mu)) \\
=\operatorname{dim}_{\operatorname{Ext}_{C(\mu)}^{n}\left(M((x s) \cdot \mu), U_{\alpha} L(y \cdot \lambda)\right)} \\
\quad-\operatorname{dim} \operatorname{Ext}_{C(\mu)}^{n+1}(M((x s) \cdot \mu), L(y \cdot \mu)) .
\end{aligned}
$$

But now, we may use Lemma 7.6 to compute

$$
\operatorname{dim} \operatorname{Ext}_{C(\mu)}^{n}\left(M((x s) \cdot \mu), U_{\alpha} L(y \cdot \lambda)\right)
$$


by taking the sum of the various $\operatorname{dim} \operatorname{Ext}_{C(\mu)}^{n}(M((x s) \cdot \mu), L(z \cdot \mu))$ with $L(z \cdot \mu)$ a composition factor of $U_{\alpha} L(y \cdot \lambda)$. We thus obtain the desired result when we note that $L((y s) \cdot \mu)$ occurs exactly once as a composition factor of $U_{\alpha} L(y \cdot \lambda)$ by Theorem 6.6 , and by Theorem 6.6 any other composition factor is of the form $L(z \cdot \mu)$ with $z<z s$ and it occurs with multiplicity $\mu(z, y)$, by Corollary 7.10 .

Corollary 7.12. Assuming the truth of Conjecture 7.4, we may compute the polynomials $\bar{P}_{x, y}(q)$ by induction on $l(x)$ as follows.

(i) If $x=1$, then $\bar{P}_{x, y}(q)=0$ for $y \neq 1$ and $\bar{P}_{x, y}(q)=1$ for $y=1$.

(ii) If $x \neq 1$, then choose a simple reflection $s$ with $x>x s$. Then in the case where $y<y s$ we have $\bar{P}_{x, y}(q)=\bar{P}_{x s, y}(q)$, and in the case where $y>y s$ we have

$$
\bar{P}_{x, y}(q)=\bar{P}_{x s, y s}(q)+\sum_{\substack{z \in W \\ z<z s}} \mu(z, y) q^{(l(z)-l(y)+1) / 2} \bar{P}_{x s, z}(q)-q \bar{P}_{x s, y}(q) \text {. }
$$

Proof. This follows immediately from Theorem 7.11 and the definition of the polynomials $\bar{P}_{x, y}(q)$.

REMARK. If we do not assume Conjecture 7.4, part (i) and the first case $y<y s$ of part (ii) still must hold, since the proof of this case relies only on Lemma 7.1 .

\section{REFERENCES}

[1] I. N. Bernstein, I. M. Gelfand and S. I. Gelfand, Differential operators on the base affine space and a study of g-modules, Lie Groups and Their Representations, Proc. Summer School on Group Representations, János Bolyai Math. Soc., Wiley, 1975, pp. 35-69.

[2] V. V. Deodhar, O. Gabber and V. G. Kac, Structure of some categories of representations of infinite-dimensional Lie algebras, Adv. in Math., 45 (1982), 92116.

[3] H. Garland and J. Lepowsky, Lie algebra homology and the Macdonald-Kac formulas, Invent. Math., 34 (1976), 37-76.

[4] W. Neidhardt, Verma module imbeddings and the Bruhat order for Kac-Moody algebras, J. Algebra, 109 (1987), 430-438.

[5] - The BGG resolution, character and denominator formulas, and related results for Kac-Moody algebras, Trans. Amer. Math. Soc., 297 (1987), 487-504.

[6] _- Local projective resolutions and translation functors for Kac-Moody algebras, Trans. Amer. Math. Soc., 305 (1988), 221-245.

[7] _ Translation to and fro over Kac-Moody algebras, Pacific J. Math., 139 (1989), 107-153. 
[8] A. Rocha-Caridi and N. Wallach, Projective modules over graded Lie Algebras I, Math. Z., 180 (1982), 151-177.

[9] D. A. Vogan, Irreducible characters of semisimple Lie groups II. The KazhdanLusztig conjectures, Duke Math. J., 46 (1979), 805-859.

[10] - Irreducible characters of semisimple Lie groups III. Proof of KazhdanLusztig conjecture in the integral case, Invent. Math., 71 (1983), 381-417.

Received September 5, 1991 and in revised form March 2, 1992.

University of Hawail-Kapiolani Community College

HoNOLULU, HI 96816 


\section{PACIFIC JOURNAL OF MATHEMATICS}

Founded by

E. F. BECKENBACH (1906-1982) F. Wolf (1904-1989)

\section{EDITORS}

\author{
V. S. VARADARAJAN \\ (Managing Editor) \\ University of California \\ Los Angeles, CA 90024-1555 \\ vsv@math.ucla.edu \\ F. Michael Christ \\ University of California \\ Los Angeles, CA 90024-1555 \\ christ@math.ucla.edu
}

\section{Herbert Clemens}

University of Utah

Salt Lake City, UT 84112

clemens@math.utah.edu

\author{
ThOMAs ENRIGHT \\ University of California, San Diego \\ La Jolla, CA 92093 \\ tenright@ucsd.edu \\ Nicholas Ercolani \\ University of Arizona \\ Tucson, AZ 85721 \\ ercolani@math.arizona.edu \\ R. FINN \\ Stanford University \\ Stanford, CA 94305 \\ finn@gauss.stanford.edu \\ VAUGHAN F. R. Jones \\ University of California \\ Berkeley, CA 94720 \\ vfr@math.berkeley.edu
}

STEVEN KeRCKHOFF

Stanford University

Stanford, CA 94305

spk@gauss.stanford.edu

Martin ScharlemanN

University of California

Santa Barbara, CA 93106

mgscharl@henri.ucsb.edu

Harold Stark

University of California, San Diego

La Jolla, CA 92093

\section{SUPPORTING INSTITUTIONS}

UNIVERSITY OF ARIZONA

UNIVERSITY OF BRITISH COLUMBIA

CALIFORNIA INSTITUTE OF TECHNOLOGY

UNIVERSITY OF CALIFORNIA

UNIVERSITY OF MONTANA

UNIVERSITY OF NEVADA, RENO

NEW MEXICO STATE UNIVERSITY

OREGON STATE UNIVERSITY
UNIVERSITY OF OREGON

UNIVERSITY OF SOUTHERN CALIFORNIA

STANFORD UNIVERSITY

UNIVERSITY OF HAWAII

UNIVERSITY OF UTAH

WASHINGTON STATE UNIVERSITY

UNIVERSITY OF WASHINGTON 


\section{PACIFIC JOURNAL OF MATHEMATICS}

Volume 159 No. $1 \quad$ May 1993

An application of the very weak Bernoulli condition for amenable groups

SCOT ROBERT ADAMS and JEFFREY EDWARD STEIF

An application of homogenization theory to harmonic analysis on solvable Lie groups of polynomial growth

G. Alexopoulos

The standard double soap bubble in $\mathbf{R}^{2}$ uniquely minimizes perimeter

Joel Foisy, Manuel Alfaro Garcia, JefFrey FARlowe

Brock, NiCKELOUS Hodges and JASON ZimbA

Pseudo regular elements and the auxiliary multiplication they induce

BARRY E. JOHNSON

A converse to a theorem of Komlós for convex subsets of $L_{1}$

CHRISTOPHER JOHN LENNARD

General Kac-Moody algebras and the Kazhdan-Lusztig conjecture

WAYNE L. NEIDHARDT

The flow space of a directed $G$-graph

WILLIAM LINDALL PASCHKE

Primitive ideals and derivations on noncommutative Banach algebras

MARK PHILLIP THOMAS

Equivariant Nielsen numbers

PETER N-S WONG

Volumes of tubular neighbourhoods of real algebraic varieties

RichaRd ALEXANDER WONGKEW

The intrinsic group of Majid's bicrossproduct Kac algebra 